\title{
COHESIVENESS IN PROMISE PROBLEMS *
}

\author{
Ulrike Brandt ${ }^{1}$ And Hermann K.-G. Walter ${ }^{1}$
}

\begin{abstract}
Promise problems have been introduced in 1985 by S. Even e.a. as a generalization of decision problems. Using a very general approach we study solvability and unsolvability conditions for promise problems of set and language families. We show, that cores of unsolvability are completely determined by partitions of cohesive sets. We prove the existence of cores in unsolvable promise problems assuming certain closure properties for the given set family. Connections to immune sets and complexity cores are presented. Furthermore, results about cohesiveness with respect to the language families from the Chomsky hierarchy are given.
\end{abstract}

Mathematics Subject Classification. 68Q45.

\section{INTRODUCTION}

In 1985 Even, Selman and Yacobi [4] introduced the concept of promise problems as a generalization of decision problems. A promise problem consists of a pair of disjoint sets $A$ and $B$ with $A, B \subseteq S$ and a given set family $\mathcal{F} \subseteq \mathbf{2}^{S}$, where $S$ is some basic (usually infinite) set. $(A, B)$ is solvable for $\mathcal{F}$ if a $Q \subseteq S$ exists with $Q \in \mathcal{F}$ and $Q^{\mathbf{c}} \in \mathcal{F}$ and $A \subseteq Q$ and $B \subseteq Q^{\mathbf{c}}$, where $Q^{\mathbf{c}}$ is the complement of $Q$ in $S$. In the case $B=A^{\mathbf{c}}(A, B)$ is a decision problem. In applications $S=X^{*}$, where $X$ is a finite nonempty alphabet and $\mathcal{F}=\mathcal{L}$ is a language family or a complexity class $\mathcal{F}=\mathcal{C}$. From an algorithmic point of view considering a promise problem $(A, B)$ an algorithm may only produce a Yes-answer for all instances $x \in A$ and a No-answer for all $x \in B$, while no decisive answer is expected for $x \notin A \cup B$. Solvability of promise problems can be linked to the existence of approximation or "special case" algorithms (see [3]). Thus with respect to complexity of algorithms a more refined

Keywords and phrases. Promise problems, set and language families, cores of unsolvability, complexity cores, cohesive sets.

* We acknowledge seminal discussions with Martin Ziegler.

1 Fachbereich Informatik, Technische Universität Darmstadt, Germany.

hermann-walter@gmx.de 
look than for decision problems is possible. Promise problems have been considered for various fields of algorithmic computations. Especially, some decision problems which are difficult to solve allow efficient algorithm once they are weakened to a promise problem. The reader can find an overview in [5]. Looking at the theory of recursive functions [8], the separation principle is a precursor of the concept of promise problems. Furthermore, we can use the notion of cohesive sets, also known in the theory of recursive functions, by an appropriate generalization. This turns out to be the characterizing indicator for (un-)solvability of promise problems. It was well-known, that only set-theoretic arguments can be used in dealing with these concepts. We mention especially the theorem of Dekker-Myhill [8] which asserts the existence of cohesive sets under very weak conditions. Our main results are a theorem about the existence of unsolvability cores for an unsolvable promise problem $(A, B)$ and the characterization of unsolvability cores via cohesiveness of $A \cup B$. The latter enables us, to study the influence of closure operations on the unsolvability of promise problems. Though the existence of cohesive sets is guaranteed under very mild conditions, it is quite difficult, to exhibit cohesive languages with nice properties. We determine cohesive sets and noncohesive sets for language families from the Chomsky hierarchy and for families given by number theoretic properties. Especially, we prove a structure result for alphabets $X$ with two or more letters. For some special cases we can at least assert the existence of recursive cohesive languages. Using results from [2], the connection to complexity cores gives a similar result for recursive language families and complexity classes. We assume the reader to be familiar with the theory of recursive functions and sets (see $[3,8,9]$ ) and standard theory of formal languages (see $[6,7])$.

Our study of promise-problems was proposed to us by Ziegler who raised the question answered in Theorem 6.14.

\section{SET - AND LANGUAGE FAMILIES - BASIC NOTATIONS AND RESULTS}

In the following a basic set $S$ is given and we assume for set families $\mathcal{F} \subseteq \mathbf{2}^{S}$. Moreover, sets $A, A^{\prime}, B, B^{\prime}, C, \ldots, Q, \ldots$ are always subsets of $S$ and singletons $\{s\}$ are identified with $s$. We mainly deal with denumerable set families $\mathcal{F}$; i.e. a function $\mathbf{e}_{\mathcal{F}}: \mathbb{N}_{0} \rightarrow \mathbf{2}^{S}$ with $\mathbf{e}_{\mathcal{F}}\left(\mathbb{N}_{0}\right)=\mathcal{F}$ exists (enumeration of $\mathcal{F}$ ). Consider the boolean operations union, intersection and complementation in connection with set families $\mathcal{F}$. The boolean operations can be lifted to binary operations between set families $\mathcal{F}_{1}$ and $\mathcal{F}_{2}$ and unary operations for $\mathcal{F}$. Define $\mathcal{F}_{1} \oplus \mathcal{F}_{2}=\{A \cup B \mid A \in$ $\mathcal{F}_{1}$ and $\left.B \in \mathcal{F}_{2}\right\}, \mathcal{F}_{1} \odot \mathcal{F}_{2}=\left\{A \cap B \mid A \in \mathcal{F}_{1}\right.$ and $\left.B \in \mathcal{F}_{2}\right\}$ and the closure operations

$$
\begin{aligned}
& \mathcal{F}^{\mathbf{u}}=\left\{A_{1} \cup \ldots \cup A_{n} \mid n \geq 1, A_{i} \in \mathcal{F} \text { for } 1 \leq i \leq n\right\} \text { (union), } \\
& \mathcal{F}^{\mathbf{s}}=\left\{A_{1} \cap \ldots \cap A_{n} \mid n \geq 1, A_{i} \in \mathcal{F} \text { for } 1 \leq i \leq n\right\} \text { (intersection), } \\
& \mathcal{F}^{\mathbf{c o}}=\left\{A^{\mathbf{c}} \mid A \in \mathcal{F}\right\}, \mathcal{F}^{\mathbf{c c}}=\mathcal{F} \cup \mathcal{F}^{\mathbf{c o}},(\text { complementation) and } \\
& \left.\mathcal{F}^{\mathbf{b}}=\left(\left(\mathcal{F}^{\mathbf{c c}}\right)^{\mathbf{s}}\right)^{\mathbf{u}} \text { (boolean closure }\right) .
\end{aligned}
$$


Moreover, we will frequently use $\mathcal{F}^{\mathrm{dc}}=\mathcal{F} \cap \mathcal{F}^{\text {co }}$.

Note, that $\left(\mathcal{F}^{\mathbf{u}}\right)^{\mathbf{s}}=\left(\mathcal{F}^{\mathbf{s}}\right)^{\mathbf{u}}($ distributivity $),\left(\mathcal{F}^{\mathbf{c o}}\right)^{\mathbf{u}}=\left(\mathcal{F}^{\mathbf{s}}\right)^{\mathbf{c o}}($ deMorgan $)$, $\left(\mathcal{F}^{\mathbf{c c}}\right)^{\mathbf{d c}}=\mathcal{F}^{\mathbf{c c}}$ and $\left(\mathcal{F}^{\mathbf{c o}}\right)^{\mathbf{c o}}=\mathcal{F}$. There are numerous (mostly trivial) relations between these operations, for example.

Proposition 2.1. Let $\mathcal{F}_{1}, \mathcal{F}_{2} \subseteq \mathscr{2}^{S}$.

(1) $\mathcal{F}_{1} \oplus \mathcal{F}_{2} \subseteq \mathcal{F}_{1} \Rightarrow \mathcal{F}_{1} \oplus \mathcal{F}_{2}^{u} \subseteq \mathcal{F}_{1}$ and $\mathcal{F}_{1}^{u} \oplus \mathcal{F}_{2} \subseteq \mathcal{F}_{1}^{u}$

(2) $\mathcal{F}_{1} \odot \mathcal{F}_{2}^{c o} \subseteq \mathcal{F}_{1} \Rightarrow \mathcal{F}_{1} \odot\left(\mathcal{F}_{2}^{c o}\right)^{u} \subseteq \mathcal{F}_{1}^{u}$ and $\mathcal{F}_{1}^{u} \odot \mathcal{F}_{2}^{c o} \subseteq \mathcal{F}_{1}^{u}$.

In the following we frequently use the combined operation of variation of $\mathcal{F}$ by $\mathcal{V}$ defined by $\mathcal{F} \pm \mathcal{V}=\mathcal{F} \oplus \mathcal{V} \cup \mathcal{F} \odot \mathcal{V}^{\text {co }}$.

Proposition 2.2. Let $\mathcal{F}, \mathcal{V} \subseteq 2^{S}$ with $\mathcal{V} \neq \varnothing$ and $\mathcal{F} \pm \mathcal{V} \subseteq \mathcal{F}$.

(1) $\varnothing, S \in \mathcal{F} \Leftrightarrow \mathcal{V}^{c c} \subseteq \mathcal{F}$.

(2) $\mathcal{F}^{c c} \pm \mathcal{V} \subseteq \mathcal{F}^{c c}, \mathcal{F}^{u} \pm \mathcal{V} \subseteq \mathcal{F}^{u}, \mathcal{F}^{s} \pm \mathcal{V} \subseteq \mathcal{F}^{s}$ and $\mathcal{F}^{b} \pm \mathcal{V} \subseteq \mathcal{F}^{b}$.

(3) $\mathcal{F} \pm \mathcal{V}^{u} \subseteq \mathcal{F}$

In the case $\mathcal{V}=\operatorname{fin}(S)=\{A \subseteq S \mid A$ finite $\}$, the condition $\mathcal{F} \pm \operatorname{fin}(S) \subseteq \mathcal{F}$ is just the closure under finite variation. Note that, $\operatorname{fin}(S)^{\mathbf{c c}}=\left(\operatorname{fin}(S)^{\mathbf{c c}}\right)^{\mathbf{b}}$ and $\mathcal{F} \odot \boldsymbol{f i n}(S) \subseteq \boldsymbol{f i n}(S)$. By Proposition 2.2(2) $\mathcal{F}^{\mathbf{c c}}, \mathcal{F}^{\mathbf{u}}, \mathcal{F}^{\mathbf{s}}, \mathcal{F}^{\mathbf{b}}$ are closed under finite variation, if $\mathcal{F}$ is closed under finite variation.

Consider the case $S=X^{*}$, where $X$ is a nonempty, finite alphabet and $X^{*}$ is the free monoid over $X$. As usual $L \subseteq X^{*}$ is called a language and $\mathcal{L} \subseteq \mathbf{2}^{X^{*}}$ a language family. The elements of $X^{*}$ are the words $w=x_{1} \ldots x_{n}\left(x_{i} \in X\right.$ for $\left.1 \leq i \leq n\right)$ and the empty word $\mathbf{1}$. The length of $w$ is $|w|=n$ and $|\mathbf{1}|=0$. Concatenation " $w v$ " of words is the monoid operation with identity 1 . The operation can be lifted to $2^{X^{*}}$. For $L_{1,2}$ the complex product is defined by $L_{1} L_{2}=\left\{w_{1} w_{2} \mid w_{1} \in L_{1}, w_{2} \in L_{2}\right\}$. $L^{*}$ is the generated submonoid.

On $X^{*}$ we can define various (partial) orderings. The following two ones are of interest to us. Define for $v, w \in X^{*}$ the prefix-ordering by $w \leq v$ (pref) $\Leftrightarrow$ $v \in w X^{*}$. Given a bijection ord $: X \rightarrow[0 \ldots b-1](b=\#(X))$ we can define also a well-ordering lex $_{\text {ord }}$ by $w \leq v\left(\mathbf{l e x}_{\text {ord }}\right)$ if and only if $|w|<|v|$ or $\forall u \in$ $X^{*}, x, y \in X: u x \leq w$ (pref) and $u y \leq v($ pref $) \Rightarrow \operatorname{ord}(x) \leq \boldsymbol{o r d}(y)$. Since lex $_{\text {ord }}$ is a well-ordering, we can define a successor function succ $_{\text {ord }}$ for $w \in X^{*}$ by $\boldsymbol{s u c c}_{\text {ord }}(w)=\min \left\{v \in X^{*} \mid w \neq v\right.$ and $\left.w \leq v\left(\operatorname{lex}_{\text {ord }}\right)\right\}$, where the minimum is taken with respect to $\operatorname{lex}_{\text {ord }}$. Then $\operatorname{char}^{*}(i)=\boldsymbol{s u c c}_{\text {ord }}^{i}(\mathbf{1})(i \geq 0)$ defines a bijection char $^{*}: \mathbb{N}_{0} \rightarrow X^{*}$.

The language families from the Chomsky hierarchy are $\mathcal{L}_{\text {r.e. }}(X)$ (recursively enumerable languages), $\mathcal{L}_{\mathbf{c s}}(X)$ (contextsensitive languages), $\mathcal{L}_{\mathbf{c f}}(X)$ (contextfree languages) and $\mathcal{L}_{\text {reg }}(X)$ (regular languages). All these families are closed under variation by $\mathcal{L}_{\text {reg }}(X)$. By encoding the generating grammars we find special enumerations $\mathbf{e}_{\mathbf{r} . \mathbf{e}}, \mathbf{e}_{\mathbf{c s}}, \mathbf{e}_{\mathbf{c f}}$ and $\mathbf{e}_{\mathbf{r e g}}$ of the corresponding language family. With these enumerations we can study decision problems and constructions for the descriptional devices (grammars). Look for example at the word-problem for $\mathcal{L}_{\mathbf{c s}}(X)$. Using $0,1 \in \mathbb{N}_{0}$ as truth values, define the predicate 
$\boldsymbol{w o r d}_{\mathbf{c s}}(i, j)=\operatorname{char}^{*}(i) \in \mathbf{e}_{\mathbf{c s}}(j) "(i, j \geq 0)$. Then $\boldsymbol{w o r d}_{\mathbf{c s}} \in \boldsymbol{r e c}_{2}$, where $\boldsymbol{r e c}_{n}(n \geq 0)$ is the set of $n$-ary recursive functions. In the case of complexity classes $\mathcal{C}$ we can find enumerations $\mathbf{e}_{\mathcal{C}}$, such that $\boldsymbol{w o r d}_{\mathcal{C}}(i, j)=" \operatorname{char}^{*}(i) \in \mathbf{e}_{\mathcal{C}}(j)$ " $(i, j \geq 0)$ is recursive. Here we have to use as descriptional devices Turing machines with reasonable resource bounds (time-/space-constructibility (see [3])). More general, let $\boldsymbol{w o r d}_{\mathbf{e}}(i, j)=" \operatorname{char}^{*}(i) \in \mathbf{e}(j) "(i, j \geq 0)$ for any $\mathbf{e}: \mathbb{N}_{0} \rightarrow \mathbf{2}^{X^{*}}$. We call $\mathbf{e}$ $W P$-recursive if and only if $\boldsymbol{w o r d}_{\mathbf{e}} \in \boldsymbol{r e c}_{2}$. A language family $\mathcal{L}$ is $W P$-recursive, if a WP-recursive enumeration e of $\mathcal{L}$ exists. In this case $\mathcal{L} \subset \mathcal{L}_{\text {rec }}(X)=\mathcal{L}_{\text {r.e. }}(X)^{\text {dc }}$ (recursive languages). Note, that for WP-recursive families a uniform solution for the word-problem exists. Complexity classes are WP-recursive and closed under variation by $\mathcal{L}_{\text {reg }}(X)$.

Considering $\mathcal{L}_{\text {reg }}(X)$ and $\mathcal{L}_{\text {cf }}(X)$ we obtain by the classical decidability results, that the predicates $\boldsymbol{e m p t y}_{\mathbf{c f}}(i)=" \mathbf{e}_{\mathbf{c f}}(i)=\emptyset "$ and finite $_{\mathbf{c f}}(i)=$ "e $\mathbf{e}_{\mathbf{c f}}(i) \in$ $\boldsymbol{f i n}\left(X^{*}\right) "(i \geq 0)$ are recursive. Moreover, $f_{\text {sect }} \in \boldsymbol{r e c}_{2}$ (intersection with regular sets) and $f_{\text {comp }} \in$ rec $_{1}$ (complementation of regular sets) exist with $\mathbf{e}_{\mathbf{c f}}(i) \cap \mathbf{e}_{\mathbf{r e g}}(j)=\mathbf{e}_{\mathbf{c f}}\left(f_{\text {sect }}(i, j)\right)$ and $\mathbf{e}_{\mathbf{r e g}}(i)^{\mathbf{c}}=\mathbf{e}_{\mathbf{r e g}}\left(f_{\text {comp }}(i)\right)(i, j \geq$ $0)$. Using all these functions, we find $\operatorname{incl}(i, j)=" \mathbf{e}_{\mathbf{c f}}(i) \subseteq \mathbf{e}_{\mathbf{r e g}}(j) "=$ $\boldsymbol{e m p t y}_{\mathbf{c f}}\left(f_{\text {sect }}\left(i, f_{\text {comp }}(j)\right)\right)(i, j \geq 0)$, hence $\boldsymbol{i n c l} \in \boldsymbol{r e c}_{2}$.

In the following, at various points we are faced with marking languages at the left, i.e. we have to consider the left translation " $w L$ ".

Proposition 2.3. For all languages $L, L_{1,2}$ and $w \in X^{*}$ :

(1) $w\left(L_{1} \cup L_{2}\right)=w L_{1} \cup w L_{2}$ and $w\left(L_{1} \cap L_{2}\right)=w L_{1} \cap w L_{2}$,

(2) $w L^{c}=(w L)^{c} \cap w X^{*}$ and $(w L)^{c}=w L^{c} \cup\left(w X^{*}\right)^{c}$.

For a language family $\mathcal{L}$ define $\mathcal{L}^{\text {ltr }}=\left\{w L \mid w \in X^{*}, L \in \mathcal{L}\right\}$ (left translation). $\mathcal{L}^{\text {ltr }}$ is another closure operation and $\mathcal{L}=\mathcal{L}^{\text {ltr }}$ if and only if $x L \in \mathcal{L}$ for any $x \in X$. Moreover, a companion to Proposition 2.2(1) (with $\mathcal{V}=\operatorname{fin}\left(X^{*}\right)$ ) holds.

Proposition 2.4. Let $\mathcal{L}=\mathcal{L}^{u}=\mathcal{L}^{\text {ltr }}$. Then fin $\left(X^{*}\right)^{c c} \subseteq \mathcal{L}$ if and only if $\varnothing, 1, X^{*} \in \mathcal{L}$.

Proof. Let $\varnothing, \mathbf{1}, X^{*} \in \mathcal{L}$. Since $w=w \mathbf{1}$ and $\mathcal{L}=\mathcal{L}^{\text {ltr }}$, singletons are in $\mathcal{L}$. But then $\operatorname{fin}\left(X^{*}\right) \subseteq \mathcal{L}$, because $\mathcal{L}=\mathcal{L}^{\mathbf{u}}$. Let $X^{k}=\left\{w \in X^{*}|| w \mid=k\right\}(k \geq 0)$. Then $X^{k}$ is finite. Hence $X^{k} X^{*}$ is the finite union of sets $w X^{*}$, i.e. $X^{k} X^{*} \in \mathcal{L}^{\mathbf{u}}=\mathcal{L}$. Let $L \in \boldsymbol{f i n}\left(X^{*}\right)$ and $k>\max \{\mid w \| w \in L\}$, then $L^{\mathbf{c}}=\left(\left(X^{k} X^{*}\right)^{\mathbf{c}} \backslash L\right) \cup X^{k} X^{*}$. Note that $\left(\left(X^{k} X^{*}\right)^{\mathbf{c}} \backslash L\right)$ is finite and therefore an element of $\mathcal{L}$ as shown before. In total $L^{c} \in \operatorname{fin}\left(X^{*}\right) \oplus \mathcal{L} \subseteq \mathcal{L}^{\mathbf{u}}=\mathcal{L}$, i.e. $\operatorname{fin}\left(X^{*}\right)^{\mathbf{c o}} \subseteq \mathcal{L}$.

In connection with boolean operations we get

Lemma 2.5. If $\mathcal{L}=\mathcal{L}^{\text {ltr }}$, then

(1) $\left(\mathcal{L}^{u}\right)^{l t r}=\mathcal{L}^{u}$ and $\left(\mathcal{L}^{s}\right)^{l t r}=\mathcal{L}^{s}$.

(2) $\left(\mathcal{L}^{c c}\right)^{l t r}=\mathcal{L}^{c c}$, if additionally $\mathcal{L} \pm \mathcal{L}_{\text {reg }}(X) \subseteq \mathcal{L}$. 
Proof.

(1) By Proposition 2.3(1): $\left(\mathcal{L}^{\mathbf{u}}\right)^{\text {ltr }} \subseteq\left(\mathcal{L}^{\text {ltr }}\right)^{\mathbf{u}}=\mathcal{L}^{\mathbf{u}}$. Analogously, $\left(\mathcal{L}^{\mathbf{s}}\right)^{\mathbf{l t r}} \subseteq \mathcal{L}^{\mathbf{s}}$.

(2) Since $w X^{*} \in \mathcal{L}_{\text {reg }}(X)$ for all $w \in X^{*}$, we get by our assumption, Proposition 2.3(2) and Proposition 2.2(2) $\left(\mathcal{L}^{\text {co }}\right)^{\text {ltr }} \subseteq\left(\mathcal{L}^{\text {ltr }}\right)^{\text {co }} \odot \mathcal{L}_{\text {reg }}(X) \subseteq\left(\mathcal{L}^{\text {ltr }}\right)^{\text {cc }} \pm$ $\mathcal{L}_{\text {reg }}(X)=\mathcal{L}^{\text {cc }} \pm \mathcal{L}_{\text {reg }}(X) \subseteq \mathcal{L}^{\text {cc }}$. This shows $\left(\mathcal{L}^{\text {cc }}\right)^{\text {ltr }} \subseteq \mathcal{L}^{\text {cc }}$.

Next we look at the inverse of left translations (removing left markers).

Definition 2.6. $\mathcal{L}$ is ltr-cancellative if and only if for all $L \subseteq X^{*}$ and $w \in X^{*}$ : $w L \in \mathcal{L} \Rightarrow L \in \mathcal{L}$.

Proposition 2.7. If $\mathcal{L}$ is ltr-cancellative, then

(1) $\mathcal{L}^{u}$ is ltr-cancellative

(2) $\mathcal{L}^{\text {co }}$ is ltr-cancellative, if additionally $\mathcal{L} \pm \mathcal{L}_{\text {reg }}(X) \subseteq \mathcal{L}$.

Proof. (1) Let $w L=L_{1} \cup \ldots \cup L_{n}$ with $L_{i} \in \mathcal{L}$ for $1 \leq i \leq n$. Then each $L_{i} \subseteq w X^{*}$, i.e. $L_{i}=w L_{i}^{\prime}$. Since $\mathcal{L}$ is ltr-cancellative, $L_{i}^{\prime} \in \mathcal{L}$. But then $L=$ $L_{1}^{\prime} \cup \ldots \cup L_{n}^{\prime} \in \mathcal{L}^{\mathbf{u}}$. (2) If $w L \in \mathcal{L}^{\mathbf{c o}}$, then $(w L)^{\mathbf{c}} \in \mathcal{L}$. Since $(w L)^{\mathbf{c}}=w L^{\mathbf{c}} \cup\left(w X^{*}\right)^{\mathbf{c}}$ by Proposition 2.3(2) and $\mathcal{L} \pm \mathcal{L}_{\text {reg }}(X) \subseteq \mathcal{L}$, we get $w L^{\mathbf{c}} \in \mathcal{L}$ and therefore $L^{\mathbf{c}} \in \mathcal{L}$. Hence $L \in \mathcal{L}^{\text {co }}$, i.e. $\mathcal{L}^{\text {co }}$ is ltr-cancellative.

All families from the Chomsky hierarchy and all complexity classes are ltrcancellative and closed under left translation.

\section{Cohesiveness}

Definition 3.1. $A$ is $\mathcal{F}$-cohesive $(A \in \operatorname{cohesive}(\mathcal{F}))$ if and only if $A \notin \operatorname{fin}(S)$ and for any $B \in \mathcal{F}^{\mathrm{dc}}:\left(A \cap B \notin \operatorname{fin}(S) \Rightarrow A \cap B^{\mathbf{c}} \in \operatorname{fin}(S)\right)$.

Remark 3.2. The definition of cohesiveness given in Section 12.3 of [8] is equivalent to $\mathcal{L}_{\text {r.e. }}(X)^{\text {cc }_{\text {-cohesiveness. }}}$

Directly from the definition we get

\section{Proposition 3.3.}

(1) $\operatorname{cohesive}(\mathcal{F})=\operatorname{cohesive}\left(\mathcal{F}^{c o}\right)=\operatorname{cohesive}\left(\mathcal{F}^{d c}\right)$

(2) $\mathcal{F}_{1} \subseteq \mathcal{F}_{2} \Rightarrow \operatorname{cohesive}\left(\mathcal{F}_{2}\right) \subseteq \operatorname{cohesive}\left(\mathcal{F}_{1}\right)$.

Next we study the influence of closure operations on cohesiveness. Especially, we discuss boolean operations and closure under finite variation and left translation.

Lemma 3.4. If $\mathcal{F}=\mathcal{F}^{c c}$, then cohesive $(\mathcal{F})=\operatorname{cohesive}\left(\mathcal{F}^{b}\right)$.

Proof. By our assumption $\mathcal{F}^{\mathbf{b}}=\left(\mathcal{F}^{\mathbf{s}}\right)^{\mathbf{u}}$. Consider $A \in \operatorname{cohesive}(\mathcal{F})$ and $B \in \mathcal{F}^{\mathbf{b}}$ with $A \cap B \notin \operatorname{fin}(S)$. Then $B=B_{1} \cup \ldots \cup B_{n}$ with $B_{i} \in \mathcal{F}^{\mathbf{s}}$ for $1 \leq i \leq n$. Now, $A \cap\left(B_{1} \cup \ldots \cup B_{n}\right)=\left(A \cap B_{1}\right) \cup \ldots \cup\left(A \cap B_{n}\right) \notin \operatorname{fin}(S)$. But then $C=B_{j}$ exists with $A \cap C \notin \operatorname{fin}(S)$. Since $C \subseteq B$, we know that $A \cap B^{\mathbf{c}} \subseteq A \cap C^{\mathbf{c}}$. Hence, if $A \cap C^{\mathbf{c}} \in \operatorname{fin}(S)$, then $A \cap B^{\mathbf{c}} \in \operatorname{fin}(S)$. 
Since $C \in \mathcal{F}^{\mathrm{s}}, C=C_{1} \cap \ldots \cap C_{m}$ with $C_{i} \in \mathcal{F}$ for $1 \leq i \leq m$. Furthermore, $A \cap C \notin \operatorname{fin}(S)$, so that for every i, $A \cap C_{i} \notin \operatorname{fin}(S)$. But then, by the cohesiveness of $A, A \cap C_{i}^{\mathbf{c}} \in \operatorname{fin}(S)$ for $1 \leq i \leq m$ and therefore $A \cap C^{\mathbf{c}}=A \cap\left(C_{1} \cap \ldots \cap C_{m}\right)^{\mathbf{c}}=$ $A \cap\left(C_{1}^{\mathbf{c}} \cup \ldots \cup C_{m}^{\mathbf{c}}\right)=\left(A \cap C_{1}^{\mathbf{c}}\right) \cup \ldots \cup\left(A \cap C_{n}^{\mathbf{c}}\right) \in \operatorname{fin}(S)$.

Proposition 3.5. cohesive $(\mathcal{F})$ is closed under finite variation.

Proof. Consider $A \in \operatorname{cohesive}(\mathcal{F}), C \in \operatorname{fin}(S)$ and some $B \in \mathcal{F}$. Assume that $(A \cup C) \cap B=(A \cap B) \cup(C \cap B) \notin \operatorname{fin}(S)$. Since $C \cap B \in \operatorname{fin}(S), A \cap B \notin \operatorname{fin}(S)$ and therefore $A \cap B^{\mathbf{c}} \in \operatorname{fin}(S)$ due to the cohesiveness of $A$. Since $C \cap B^{\mathbf{c}} \in \operatorname{fin}(S)$ as well, $(A \cup C) \cap B^{\mathbf{c}}=\left(A \cap B^{\mathbf{c}}\right) \cup\left(C \cap B^{\mathbf{c}}\right) \in \operatorname{fin}(S)$.

In the second step, assume that $\left(A \cap C^{\mathbf{c}}\right) \cap B=(A \cap B) \cap C^{\mathbf{c}} \notin \operatorname{fin}(S)$. Then $A \cap B \notin \operatorname{fin}(S)$, i.e. $A \cap B^{\mathbf{c}} \in \operatorname{fin}(S)$, because $A$ is $\mathcal{F}$-cohesive. But then $\left(A \cap C^{\mathbf{c}}\right) \cap B^{\mathbf{c}}=\left(A \cap B^{\mathbf{c}}\right) \cap C^{\mathbf{c}} \in \operatorname{fin}(S)$.

For $S=X^{*}$ and left translation we can show

Lemma 3.6. If $\mathcal{L}$ is ltr-cancellative, $\mathcal{L}=\mathcal{L}^{\text {ltr }}$ and $\mathcal{L} \pm \mathcal{L}_{\text {reg }}(X) \subseteq \mathcal{L}$, then for any $L \in \mathcal{L}, w \in X^{*}: L \in \operatorname{cohesive}(\mathcal{L})$ if and only if $w L \in \operatorname{cohesive}(\mathcal{L})$.

Proof. Suppose $L \in$ cohesive $(\mathcal{L})$, i.e. $L \cap B \notin \operatorname{fin}(S)$ implies $L \cap B^{\mathbf{c}} \in \operatorname{fin}(S)$ for any $B \in \mathcal{L}$. Consider $w \in X^{*}$ and $B \in \mathcal{L}^{\text {dc }}$ with $w L \cap B \notin \operatorname{fin}(S)$. Then we have to show, that $w L \cap B^{\mathbf{c}} \in \operatorname{fin}(S)$. Clearly, $w L \cap B=w(L \cap A)$ for $A$ with $w A=B \cap w X^{*}$. Thus, $A \in \mathcal{L}$, since $\mathcal{L}$ is ltr-cancellative and closed under finite variation by regular sets. By the same arguments we get $A^{\mathbf{c}} \in \mathcal{L}$, too: To see this, observe that $w A^{\mathbf{c}}=(w A)^{\mathbf{c}} \cap\left(w X^{*}\right)$ by Proposition 1.3(2) and therefore $w A^{\mathbf{c}}=\left(B \cap w X^{*}\right)^{\mathbf{c}} \cap w X^{*}=\left(B^{\mathbf{c}} \cup\left(w X^{*}\right)^{\mathbf{c}}\right) \cap w X^{*}=B^{\mathbf{c}} \cap w X^{*}$.

Now, $w L \cap B \notin \operatorname{fin}(S)$ implies $L \cap A \notin \operatorname{fin}(S)$. Hence, $L \cap A^{\mathbf{c}} \in \operatorname{fin}(S)$ by

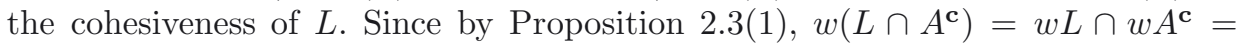
$w L \cap\left(B^{\mathbf{c}} \cap w X^{*}\right)=w L \cap B^{\mathbf{c}}, w L \cap B^{\mathbf{c}}$ must be finite, too.

Conversely, suppose $w L \in \operatorname{cohesive}(\mathcal{L})$ for some $w \in X^{*}$. Fix $B \in \mathcal{L}^{\mathrm{dc}}$ with $L \cap B \notin \operatorname{fin}(S)$. We show, that $L \cap B^{\mathbf{c}}$ is finite. Observe, that $w B \in \mathcal{L}^{\text {dc }}$ by the closure properties of $\mathcal{L}$. Now, $L \cap B \notin \operatorname{fin}\left(X^{*}\right)$ implies $w L \cap w B^{\mathbf{c}} \notin \operatorname{fin}\left(X^{*}\right)$ and therefore $w L \cap(w B)^{\mathbf{c}} \in \boldsymbol{f i n}\left(X^{*}\right)$, because $w L$ is $\mathcal{L}$-cohesive. But $w L \cap(w B)^{\mathbf{c}}=$ $w L \cap w B^{\mathbf{c}}=w\left(L \cap B^{\mathbf{c}}\right)$, i.e. $L \cap B^{\mathbf{c}} \in \operatorname{fin}\left(X^{*}\right)$.

The existence of cohesive sets for denumerable set families is guaranteed by a result of Dekker and Myhill ( $c f$. Thm. VI in Sect. 12.3 of [8]).

Theorem 3.7 (Dekker and Myhill). Let $\mathcal{F}$ be a denumerable set family. Then for any $A \notin \operatorname{fin}(S)$ there is a subset $B$ of $A$ with $B \in \operatorname{cohesive}(\mathcal{F})$.

The following fact is obvious:

Proposition 3.8. If $B \subseteq A, B \notin \operatorname{fin}(S)$ and $A \in \operatorname{cohesive}(\mathcal{F})$ then $B \in$ $\operatorname{cohesive}(\mathcal{F})$.

A natural generalization of Theorem VII(ii) in Section 12.3 of [8] is 
Lemma 3.9. If $A, B \in \operatorname{cohesive}(\mathcal{F})$ and $A \cap B \notin \operatorname{fin}(S)$ then $A \cup B \in \operatorname{cohesive}(\mathcal{F})$.

Remark 3.10. Note that the condition " $A \cap B \notin \boldsymbol{f i n}(S)$ " in Lemma 3.9 is necessary. To see this, consider $X=\{a, b\}$ and $\mathcal{L}$ satisfying the condition of Lemma 3.6. If $L \in$ cohesive $(\mathcal{L})$, then $a L, b L \in$ cohesive $(\mathcal{L})$. But $(a L \cup b L) \cap a X^{*}=a L \notin$ fin $\left(X^{*}\right)$ and $b L \subseteq(a L \cup b L) \cap\left(a X^{*}\right)^{\mathbf{c}} \notin \operatorname{fin}\left(X^{*}\right)$. Hence, $a L \cup b L \notin \operatorname{cohesive}(\mathcal{L})$.

Cohesiveness is a stronger condition than immunity for sets in connection with set families. For a set family $\mathcal{F}$ a set $A$ is defined to be $\mathcal{F}$-immune if it is infinite and has no infinite subset in $\mathcal{F}$, i.e. if $A^{\mathbf{c}} \cap B \neq \varnothing$ for any $B \in \mathcal{F} \backslash \boldsymbol{f i n}(S)$ (cf. e.g. [3,8]). Let immune $(\mathcal{F})$ denote the family of all $\mathcal{F}$-immune sets. Clearly, infinite subsets of $\mathcal{F}$-immune sets are $\mathcal{F}$-immune and immune $\left(\mathcal{F}_{2}\right) \subseteq \operatorname{immune}\left(\mathcal{F}_{1}\right)$, if $\mathcal{F}_{1} \subseteq \mathcal{F}_{2}$.

Proposition 3.11. If $\mathcal{F}$ is closed under finite variation and $A \in \operatorname{cohesive}(\mathcal{F}) \backslash \mathcal{F}$ then $A \in \operatorname{immune}(\mathcal{F})$.

Proof. Suppose $B \in \mathcal{F} \backslash \boldsymbol{f i n}(S)$ exists with $A^{\mathbf{c}} \cap B=\varnothing$. Then $A \cap B=B \notin \operatorname{fin}(S)$ and therefore $A \cap B^{\mathbf{c}} \in \operatorname{fin}(S)$, because $A$ is $\mathcal{F}$-cohesive. Since $\mathcal{F}$ is closed under finite variation $A=B \cup\left(A \cap B^{\mathbf{c}}\right) \in \mathcal{F}$ - a contradiction.

Remark 3.12. Theorem $\mathrm{V}$ in Section 12.3 of [8] shows, that any $L \in$ cohesive $\left(\mathcal{L}_{\text {r.e. }}(X)^{\text {cc }}\right)$ is not only immune but hyperimmune and even hyperhyperimmune.

Example 3.13. Let $X=\{a, b\}$. Then $A=\left\{a^{n} b^{n} \mid n>0\right\}$ is $\mathcal{L}_{\text {reg }}(X)$-immune (use the pumping lemma for $\left.\mathcal{L}_{\text {reg }}(X)\right)$, but not $\mathcal{L}_{\text {reg }}(X)$-cohesive (consider e.g. $\left.R=\left(a^{2}\right)^{*}\left(b^{2}\right)^{*}\right)$.

Example 3.14. $\mathcal{L}$-cohesive languages need not necessarily be outside of $\mathcal{L}$ :

(1) Any $A \notin \boldsymbol{f i n}(S)$ is $\boldsymbol{f i n}(S)^{\mathbf{c c}}$-cohesive, for example $X^{*}\left(\in \boldsymbol{f i n}\left(X^{*}\right)^{\mathbf{c c}}\right)$.

(2) By a theorem of Friedberg $L \in \mathcal{L}_{\text {r.e. }}(X)^{\text {co }}$ exists with $L \in$ cohesive $\left(\mathcal{L}_{\text {r.e. }}(X)^{\mathbf{c c}}\right)$ (see Thm. XI in Sect. 12.4 of [8] for details).

\section{Cohesiveness of LANGUAGeS}

We derive special results for cohesiveness with respect to language families, especially for the families from the Chomsky hierarchy and complexity classes. For all these families $\mathcal{L}$ any $\mathcal{L}$-cohesive language has a specific structural property. This property is connected to infinite words. Infinite words can be defined using pref-isotone and length-preserving functions.

Definition 4.1. $f: \mathbb{N}_{0} \rightarrow X^{*}$ is sequential if and only if for any $n \geq 0:|f(n)|=$ $n$ and $f(n) \leq f(n+1)$ (pref).

Lemma 4.2. If $\#(X)>1$ and $L \in \operatorname{cohesive}\left(\mathcal{L}_{\text {reg }}(X)\right)$, then a sequential $f_{L}: \mathbb{N}_{0} \rightarrow X^{*}$ exists with $L \backslash f_{L}(n) X^{*} \in \operatorname{fin}\left(X^{*}\right)$ for any $n \geq 0$. 
Proof. The key to the proof is the following

Assertion. If $L \in \operatorname{cohesive}\left(\mathcal{L}_{\text {reg }}(X)\right)$, then for all $u, v \in X^{*}$ with $|u|=|v|$ : $L \cap u X^{*}, L \cap v X^{*} \notin \operatorname{fin}\left(X^{*}\right)$ implies $u=v$.

Suppose the contrary, i.e. $u, v \in X^{*}$ exist with $|u|=|v|, L \cap u X^{*}, L \cap v X^{*} \notin$ $\operatorname{fin}\left(X^{*}\right)$ and $u \neq v$. Then $u X^{*} \cap v X^{*}=\varnothing$. Hence, $v X^{*} \cap L \subseteq\left(u X^{*}\right)^{\mathbf{c}} \cap L$ and therefore $\left(u X^{*}\right)^{\mathbf{c}} \cap L \notin \operatorname{fin}\left(X^{*}\right)$. Thus, $L \notin$ cohesive $\left(\mathcal{L}_{\text {reg }}(X)\right)$ - a contradiction.

Since $L \notin \operatorname{fin}\left(X^{*}\right)$, we can find to any $n \geq 0$ some $w \in X^{*}$ with $|w|=n$ and $L \cap w X^{*} \notin \operatorname{fin}\left(X^{*}\right)$. Define $f_{L}(n)=w$. By the assertion $f_{L}$ is uniquely determined. Furthermore, if $u \leq w$ (pref), then $L \cap w X^{*} \subseteq L \cap u X^{*}$. Hence, $L \cap u X^{*} \notin \operatorname{fin}\left(X^{*}\right)$ and by the assertion $f_{L}(|u|)=u$. That is, $f_{L}$ is sequential. Moreover, since $L \in$ cohesive $\left(\mathcal{L}_{\text {reg }}(X)\right), L \cap\left(f_{L}(n) X^{*}\right)^{\mathbf{c}} \in \operatorname{fin}\left(X^{*}\right)$ for all $n \geq 0$.

From the assertion in this proof we get additionally the following

Corollary 4.3. If $\#(X)>1$ and $L \in \operatorname{cohesive}\left(\mathcal{L}_{\text {reg }}(X)\right)$, then for any $L^{\prime} \subseteq L$ with $L^{\prime} \notin \operatorname{fin}\left(X^{*}\right): f_{L}=f_{L^{\prime}}$.

Proof. Suppose $n \geq 0$ exists with $f_{L}(n) \neq f_{L^{\prime}}(n)$. We know by Lemma 4.2 and Proposition 3.8 that $L^{\prime} \cap f_{L^{\prime}}(n) X^{*}, L \cap f_{L}(n) X^{*} \notin$ fin $\left(X^{*}\right)$. Furthermore, $L^{\prime} \cap$ $f_{L^{\prime}}(n) X^{*} \subseteq L \cap f_{L^{\prime}}(n) X^{*}$. Hence, $L \cap f_{L^{\prime}}(n) X^{*}$ is infinite. This is a contradiction to the above assertion.

Next, we focus our attention to $\mathcal{L}_{\text {reg }}(X)$-cohesiveness in connection with $\mathcal{L}_{\text {cf }}(X)$ and $\mathcal{L}_{\mathbf{c s}}(X)$. To $L \subseteq X^{*}$ we associate the length-language $|L|=\lambda_{X}(L)$, where $\lambda_{X}(w)=a^{|w|}\left(w \in X^{*}\right)$. Define $\mathcal{L}_{\text {lreg }}(X)=\left\{L \subseteq X^{*}|| L \mid \in \mathcal{L}_{\text {reg }}(a)\right\}$. Note that $\left\{a^{n} b^{n} a^{n} \mid n \geq 0\right\} \in \mathcal{L}_{\text {lreg }}(\{a, b\})$.

Lemma 4.4. If $L \in \mathcal{L}_{\text {lreg }}(X)$, then $L \notin \operatorname{cohesive}\left(\mathcal{L}_{\text {reg }}(X)\right)$.

Proof. $|L| \in \mathcal{L}_{\text {reg }}(a)$ and $|L| \notin \boldsymbol{f i n}\left(a^{*}\right)$, since $L \notin \boldsymbol{f i n}\left(X^{*}\right)$. By the pumping lemma for $\mathcal{L}_{\text {reg }}(a) \alpha>0$ and $\beta \geq 0$ exist with $\left(a^{\alpha}\right)^{*} a^{\beta} \subseteq|L|$. Consider $R=\left(a^{2 \alpha}\right)^{*} a^{\beta} \in$ $\mathcal{L}_{\text {reg }}(a) \backslash$ fin $\left(a^{*}\right)$. Then $R \subseteq|L|$ and $R^{\mathbf{c}} \cap|L| \notin$ fin $\left(a^{*}\right)$. But $\lambda_{X}^{-1}(R), \lambda_{X}^{-1}\left(R^{\mathbf{c}}\right) \in$ $\mathcal{L}_{\text {reg }}(X), L \cap \lambda_{X}^{-1}(R), L \cap \lambda_{X}^{-1}\left(R^{\mathbf{c}}\right) \notin \operatorname{fin}\left(X^{*}\right)$ and $\lambda_{X}^{-1}\left(R^{\mathbf{c}}\right) \subseteq \lambda_{X}^{-1}(R)^{\mathbf{c}}$.

Since $\mathcal{L}_{\text {reg }}(X) \subseteq \mathcal{L}_{\text {cf }}(X) \subseteq \mathcal{L}_{\text {lreg }}(X)$, we get immediately

Lemma 4.5. If $L \in$ cohesive $\left(\mathcal{L}_{c f}(X)\right)$, then $L \notin \mathcal{L}_{c f}(X)$.

Inspecting the "construction" from the proof of the Dekker-Myhill-theorem (Thm. VI in Sect. 12.3 of [8]) yields

Theorem 4.6. For any $L \in \mathcal{L}_{c f}(X) \backslash$ fin $\left(X^{*}\right)$ a recursive language $L^{\prime} \subseteq L$ exists with $L^{\prime} \in$ cohesive $\left(\mathcal{L}_{\text {reg }}(X)\right)$. 
Proof. We refine the proof of the Myhill-Dekker-theorem. Define inductively

$$
\begin{aligned}
& L_{0}=L, \\
& L_{n+1}=\text { if } L_{n} \cap \mathbf{e}_{\text {reg }}(n) \notin \operatorname{fin}\left(X^{*}\right) \text { then } L_{n} \cap \mathbf{e}_{\text {reg }}(n) \text { else } L_{n} \cap \mathbf{e}_{\mathbf{r e g}}(n)^{\mathbf{c}} \mathbf{f i} \\
& (n \geq 0) .
\end{aligned}
$$

Then for $n \geq 0 L_{n+1} \subseteq L_{n}$ and $L_{n} \in \mathcal{L}_{\text {cf }}(X)$. Moreover, $L_{n} \notin \operatorname{fin}\left(X^{*}\right)$ and $L_{n} \notin$ cohesive $\left(\mathcal{L}_{\text {reg }}(X)\right)$ by Lemma 4.5. Now, a function $g$ exists with $L_{g(n+1)} \subset$ $L_{g(n)} \subseteq L_{n}$. Define the function $h$ by $h(n)=\min \left\{m \mid \operatorname{char}^{*}(m) \in L_{g(n)} \backslash L_{g(n+1)}\right\}$ $(n \geq 0)$. Let $L^{\prime \prime}=\operatorname{char}^{*}\left(h\left(\mathbb{N}_{0}\right)\right)$. The above mentioned proof of Theorem VI in Section 12.3 of $[8]$ asserts $L^{\prime \prime} \in \operatorname{cohesive}\left(\mathcal{L}_{\text {reg }}(X)\right)$.

It remains to prove, that any step in this construction is computable. Let $\mathbf{e}_{\mathbf{c f}}\left(i_{0}\right)=L$. Define

$$
\begin{aligned}
& f(0)=i_{0}, \\
& f(n+1)=\text { if } \text { finite }_{\text {cf }}\left(f_{\text {sect }}(f(n), n)\right)=0 \text { then } f_{\text {sect }}(f(n), n) \\
& \text { else } f_{\text {sect }}\left(f(n), f_{\text {comp }}(n)\right) \text { fi }(n \geq 0) .
\end{aligned}
$$

Since finite $_{\mathbf{c f}}$ is recursive, $f \in \boldsymbol{r e c}_{1}$ and $\mathbf{e}_{\mathbf{c f}}(f(n))=L_{n}(n \geq 0)$. Next, consider the predicate $d$ defined by $d(n, m)="(m>n)$ and $\left(L_{n}=L_{m}\right)$ ". Then $d(n, m)=$ $"(m>n)$ and $\left(\forall 1 \leq i \leq m-n: L_{n+i}=L_{m}\right) "="(m>n)$ and $(\forall 0 \leq i \leq$ $m-n:\left(L_{n+i} \subseteq \mathbf{e}_{\text {reg }}(n+i)\right)$ or $\left.\left(L_{n+i} \subseteq \mathbf{e}_{\text {reg }}(n+i)\right)^{\mathbf{c}}\right)$ ". Using $\boldsymbol{i n c l}$ and $f_{\text {comp }}$ we get $d \in \operatorname{rec}_{2}$. By this the function $g^{\prime}(n)=\min \{m \mid(m>n)$ and $d(f(n), m)=0\}$ is recursive, as well. Now, with the help of $f, g^{\prime}$ the function $g$ from above can be defined by $g(0)=f(0), g(n)=f\left(g^{\prime}(n-1)\right)(n>0)$, i.e. $g \in$ rec $_{1}$, since $f, g^{\prime} \in$ $\boldsymbol{r e c}_{1}$. Since $w(m, n)=" \operatorname{char}^{*}(m) \in L_{g(n)} \backslash L_{g(n+1)} "=\boldsymbol{w o r d}_{\mathbf{c s}}(m, f(g(n)))$ is a recursive function, $h(n)=\min \left\{m \mid \operatorname{char}^{*}(m) \in L_{g(n)} \backslash L_{g(n+1)}\right\} \in \boldsymbol{r e c}_{1}$ and we can conclude that $L^{\prime \prime}=\operatorname{char}^{*}\left(h\left(\mathbb{N}_{0}\right)\right) \in \mathcal{L}_{\text {r.e. }}(X)$. But then an infinite $L^{\prime} \subseteq L^{\prime \prime}$ exists with $L^{\prime} \in \mathcal{L}_{\text {rec }}(X)$. Since $L^{\prime \prime} \in \operatorname{cohesive}\left(\mathcal{L}_{\text {reg }}(X)\right), L^{\prime} \in \operatorname{cohesive}\left(\mathcal{L}_{\text {reg }}(X)\right)$, too.

Dealing with $\mathcal{L}_{\mathbf{c s}}(X)$ we can use number-theoretic considerations. In the case $X=\{a\}$ we know $\mathcal{L}_{\text {reg }}(X)=\mathcal{L}_{\mathbf{c f}}(X)$ and can use the pumping lemma for regular sets.

Lemma 4.7 (number-problems). Let $X=\{a\}$.

(1) $L_{\exp }=\left\{a^{2^{n}} \mid n>0\right\} \notin$ cohesive $\left(\mathcal{L}_{\text {reg }}(X)\right)$ and $L_{\exp } \in \mathcal{L}_{c s}(X)$.

(2) $L_{\mathrm{fac}}=\left\{a^{n !} \mid n>0\right\} \in \operatorname{cohesive}\left(\mathcal{L}_{\text {reg }}(X)\right)$ and $L_{\mathrm{fac}} \in \mathcal{L}_{c s}(X)$.

Proof.

(1) Clearly, $2^{2 k} \boldsymbol{m o d} 3=(3+1)^{k} \bmod 3=1$ Hence $2^{2 k+1} \boldsymbol{m o d} 3=$ $2\left(2^{2 k} \bmod 3\right) \bmod 3=2$. By this $\left\{a^{2^{2 k}} \mid k \geq 0\right\} \subseteq a\left(a^{3}\right)^{*}=R$, while $\left\{a^{2^{2 k+1}} \mid k \geq 0\right\} \subseteq R^{\mathbf{c}}$. In total $L_{\exp } \notin$ cohesive $\left(\mathcal{L}_{\text {reg }}(X)\right)$, because $L_{\exp } \cap$ $R, L_{\exp } \cap R^{\mathbf{c}} \notin \operatorname{fin}\left(a^{*}\right)$. 
(2) Consider $R \in \mathcal{L}_{\text {reg }}(X)$ with $L_{\text {fac }} \cap R \notin \boldsymbol{f i n}\left(a^{*}\right)$. Using the pumping lemma for $\mathcal{L}_{\text {reg }}(X) \alpha>0$ and $\beta$ exist with $L_{\text {fac }} \cap a^{\beta}\left(a^{\alpha}\right)^{*} \notin$ fin $\left(a^{*}\right)$ and $a^{\beta}\left(a^{\alpha}\right)^{*} \subseteq R$. Due to the infinity of the intersection we can find some $y \geq \max (\alpha, \beta)$, such that $\alpha x+\beta=y$ ! for some $x \in \mathbb{N}_{0}$. Since $y \geq \alpha, \alpha$ divides $y$ !. Hence, $\beta$ is an integer multiple of $\alpha$, too and we get $\alpha x+\beta=\alpha\left(x+\beta^{\prime}\right)=y$ ! for some $\beta^{\prime} \leq y$. But then for any $y^{\prime} \geq y$ a $z$ exists with $\alpha\left(z+\beta^{\prime}\right)=y^{\prime}$ !, namely $z=\left(x+\beta^{\prime}\right) \frac{y^{\prime} !}{y !}-\beta^{\prime}$, and we get in total $L_{\mathrm{fac}} \cap a^{\beta}\left(a^{\alpha}\right)^{*}=L_{\mathrm{fac}} \backslash C$ for some finite set $C$ and therefore $L_{\mathrm{fac}} \cap R^{\mathbf{c}} \in \operatorname{fin}\left(a^{*}\right)$.

A result similar to Lemma 4.5 is

Proposition 4.8. If $L \in \operatorname{cohesive}\left(\mathcal{L}_{\text {rec }}(X)\right)$, then $L \notin \mathcal{L}_{\text {r.e. }}(X)$.

Proof. Consider $L \in \operatorname{cohesive}\left(\mathcal{L}_{\text {rec }}(X)\right)$ and suppose that $L \in \mathcal{L}_{\text {r.e. }}(X)$. Since $L$ is infinite, $L^{\prime} \in \mathcal{L}_{\text {rec }}(X) \backslash \boldsymbol{f i n}\left(X^{*}\right)$ exists with $L^{\prime} \subseteq L$. Clearly, $L=L_{1} \cup L_{2}$ with $L_{1,2} \in \mathcal{L}_{\text {rec }}(X) \backslash \boldsymbol{f i n}\left(X^{*}\right)$ and $L_{1} \cap L_{2}=\emptyset$. But then $L \cap L_{1}=L_{1} \notin \boldsymbol{f i n}\left(X^{*}\right)$ and $L \cap L_{2} \subseteq L \cap L_{1}^{\mathbf{c}} \notin \operatorname{fin}\left(X^{*}\right)-$ a contradiction.

\section{Solvability of PROMise PROBlems}

Remember that given a set family $\mathcal{F}(A, B)$ is a promise problem, if $A \cap B=\emptyset$. With $\mathcal{F}$ we associate the set of promise problems, which are solvable with respect to $\mathcal{F}$, i.e. we consider promise $(\mathcal{F})=\left\{(A, B) \mid A \cap B=\varnothing\right.$ and $\exists Q \in \mathcal{F}^{\text {dc }}: A \subseteq$ $Q$ and $\left.B \subseteq Q^{\mathbf{c}}\right\}$. We collect some elementary facts about promise $(\mathcal{F})$, which follow more or less directly by the definition, especially by using the laws of De Morgan and distributivity.

\section{Proposition 5.1.}

(1) $(A, B) \in \operatorname{promise}(\mathcal{F}) \Leftrightarrow(B, A) \in \operatorname{promise}(\mathcal{F})$.

(2) $B^{\prime} \subseteq B$ and $(A, B) \in \operatorname{promise}(\mathcal{F}) \Rightarrow\left(A, B^{\prime}\right) \in \operatorname{promise}(\mathcal{F})$.

(3) $A \in \mathcal{F}^{\text {dc }}$ and $A \cap B=\emptyset \Rightarrow(A, B) \in \operatorname{promise}(\mathcal{F})$.

(4) $A \in \mathcal{F}^{d c} \Leftrightarrow\left(A, A^{c}\right) \in \operatorname{promise}(\mathcal{F})$.

(5) $\mathcal{F}^{\prime} \subseteq \mathcal{F} \Rightarrow$ promise $\left(\mathcal{F}^{\prime}\right) \subseteq$ promise $(\mathcal{F})$.

(6) $\operatorname{promise}(\mathcal{F})=\operatorname{promise}\left(\mathcal{F}^{c o}\right)=\operatorname{promise}\left(\mathcal{F}^{d c}\right)$.

(7) If $\mathcal{F}=\left(\mathcal{F}^{u}\right)^{s}$ then $(A, B) \in \operatorname{promise}(\mathcal{F})$ and $\left(\left(A, B^{\prime}\right) \in \operatorname{promise}(\mathcal{F}) \Rightarrow\left(A, B \cup B^{\prime}\right) \in \operatorname{promise}(\mathcal{F})\right)$.

(8) If $\mathcal{F} \pm \mathcal{V} \subseteq \mathcal{F}$ then $\forall C \in \mathcal{V}$ : $(A, B) \in \operatorname{promise}(\mathcal{F}) \Rightarrow\left(A \cup C, B \cap C^{c}\right) \in \operatorname{promise}(\mathcal{F})$.

Example 5.2. Consider $X=\{a, b\}$ and the languages $A=\left\{a^{n} b^{n} \mid n>0\right\}$ and $B=\left\{a^{n} b^{m} \mid n, m>0\right.$ and $\left.n \neq m\right\}$. Then $A, B, A^{\mathbf{c}} \in \mathcal{L}_{\mathbf{c f}}(X)$ and $B \subseteq A^{\mathbf{c}}$, hence $(A, B) \in \operatorname{promise}\left(\mathcal{L}_{\mathbf{c f}}(X)\right)$. We show, that $(A, B) \notin \operatorname{promise}\left(\mathcal{L}_{\text {reg }}(X)\right.$. Suppose the contrary, i.e. a $Q \in \mathcal{L}_{\text {reg }}(X)$ exists with $A \subseteq Q$ and $B \subseteq Q^{\mathbf{c}}$. Consider a word $w_{0}=a^{n} b^{n}$, where $n$ is sufficiently large. By the pumping lemma for regular sets $u, v, w \in X^{*}, w \neq 1$ exist with $w_{0}=u w v,|u w| \leq n$ and $u w^{k} v \in Q$ for all $k \geq 0$. 
But then $u w=a^{i}$ for some $1 \leq i \leq n$ and therefore $u w^{2} v=a^{n+|w|} b^{n} \in B \cap Q$. In total, $B \cap Q \neq \emptyset$ and we get a contradiction.

The following criterion asserts for a promise problem $(A, B)$ the existence of a nontrivial solvable subproblem.

Lemma 5.3. Let $\mathcal{V} \subseteq \mathcal{F}$ and $\mathcal{F} \pm \mathcal{V} \subseteq \mathcal{F}$. Then for all $A, B \in \mathcal{F} \backslash$ fin $(S)$ with $A \notin \operatorname{cohesive}(\mathcal{V})$ a $Q \in \mathcal{V}^{d c}$ exists, such that $A \cap Q, B \cap Q^{c} \in \mathcal{F} \backslash \operatorname{fin}(S)$ and $\left(A \cap Q, B \cap Q^{c}\right) \in$ promise $(\mathcal{V})$.

Proof. Let $A, B$ be given according to the assumption. Since $A \notin$ cohesive $(\mathcal{V}), Q \in$ $\mathcal{V}^{\text {dc }}$ exists with $A \cap Q, A \cap Q^{\mathbf{c}} \notin \operatorname{fin}(S)$. But then $B \cap Q$ or $B \cap Q^{\mathbf{c}}$ must be infinite, because otherwise $B=(B \cap Q) \cup\left(B \cap Q^{\mathbf{c}}\right) \in \operatorname{fin}(S)$. If $B \cap Q^{\mathbf{c}} \notin$ fin $(S)$, $A \cap Q, B \cap Q^{\mathbf{c}} \in \mathcal{F} \backslash \boldsymbol{f i n}(S)$ and $\left(A \cap Q, B \cap Q^{\mathbf{c}}\right) \in \operatorname{promise}(\mathcal{V})$. If $B \cap Q \notin \operatorname{fin}(S)$, $A \cap Q^{\mathbf{c}}, B \cap Q \in \mathcal{F} \backslash$ fin $(S)$ and $\left(A \cap Q^{\mathbf{c}}, B \cap Q\right) \in$ promise $(\mathcal{V})$.

It is interesting to look at $\mathcal{L}_{\text {r.e. }}(X)$. Consider a promise problem $(A, B)$ with $A \in \mathcal{L}_{\text {r.e. }}(X) \backslash \operatorname{fin}\left(X^{*}\right)$. Then $C \subseteq A$ exists with $C \in \mathcal{L}_{\text {rec }}(X) \backslash \operatorname{fin}\left(X^{*}\right)$. Hence, $(C, B) \in \operatorname{promise}\left(\mathcal{L}_{\text {rec }}(X)\right)=\operatorname{promise}\left(\mathcal{L}_{\text {r.e. }}(X)\right)$, since $B \subseteq A^{\mathbf{c}} \subseteq C^{\mathbf{c}}$. In contrast to this fact, there exists a promise problem $(A, B)$ with $A, B \in \mathcal{L}_{\text {r.e. }}(X)$ and $(A, B) \notin$ promise $\left(\mathcal{L}_{\text {r.e. }}(X)\right)$ (cf. exercise 5 -34. in [8]). But if $A, B \in \mathcal{L}_{\text {r.e. }}(X)^{\text {co }}$, then $(A, B) \in \operatorname{promise}\left(\mathcal{L}_{\text {r.e. }}(X)^{\text {co }}\right)(c f$. exercise 5 -33. in $[8])$.

We conclude this section looking at left translations.

Lemma 5.4. Let $\mathcal{L}$ be ltr-cancellative, $\mathcal{L}=\mathcal{L}^{\text {ltr }}$ and $\mathcal{L} \pm \mathcal{L}_{\text {reg }}(X) \subseteq \mathcal{L}$. Then for all $A, B \subseteq X^{*}, w \in X^{*}:(A, B) \in \operatorname{promise}(\mathcal{L}) \Leftrightarrow(w A, w B) \in \operatorname{promise}(\mathcal{L})$.

Proof. Suppose $(A, B) \in$ promise $(\mathcal{L})$. Then $Q \in \mathcal{L}^{\text {dc }}$ exists with $A \subseteq Q$ and $B \subseteq Q^{\mathbf{c}}$. But then $w A \subseteq w Q$ and $w B \subseteq w Q^{\mathbf{c}} \subseteq(w Q)^{\mathbf{c}}=w Q^{\mathbf{c}} \cup\left(w X^{*}\right)^{\mathbf{c}}$. Since $\mathcal{L}=\mathcal{L}^{\text {ltr }}$ and $\mathcal{L} \pm \mathcal{L}_{\text {reg }}(X) \subseteq \mathcal{L}$, we get $w Q,(w Q)^{\mathbf{c}} \in \mathcal{L}$.

Conversely, suppose $(w A, w B) \in$ promise $(\mathcal{L})$. Then we find a $Q \in \mathcal{L}^{\text {dc }}$ with $w A \subseteq Q$ and $w B \subseteq Q^{\mathbf{c}}$. But then $w A \subseteq Q \cap w X^{*}=w Q^{\prime}$ and $w B \subseteq Q^{\mathbf{c}} \cap w X^{*}=$ $w Q^{\prime \prime}$. Since $w Q^{\prime} \cup w Q^{\prime \prime}=\left(Q \cap w X^{*}\right) \cup\left(Q^{\mathbf{c}} \cap w X^{*}\right)=w X^{*}$ and $w Q^{\prime} \cap w Q^{\prime \prime}=\emptyset$, $Q^{\prime \prime}=Q^{\prime \mathbf{c}}$. Hence, $A \subseteq Q^{\prime}$ and $B \subseteq Q^{\prime c}$. Again by the closure properties of $\mathcal{L}$ we get $Q^{\prime}, Q^{\prime \mathbf{c}} \in \mathcal{L}$.

\section{Unsolvability of PROMISE PROBLEMS AND COHESIVENESS}

The structure of promise problems is closely related to cohesiveness, more precisely if cohesiveness can be connected to a promise problem $(A, B)$, then it is not solvable.

Theorem 6.1. If $\mathcal{F}$ is closed under finite variation and $A \cap B=\varnothing$, then the following statements are equivalent:

(1) $A, B \notin \operatorname{fin}(S)$ and $A \cup B \in \operatorname{cohesive}(\mathcal{F})$.

(2) $A, B \in \operatorname{cohesive}(\mathcal{F})$ and $(A, B) \notin \operatorname{promise}(\mathcal{F})$. 
Proof.

$(1) \Rightarrow(2)$. Let $A, B \notin \operatorname{fin}(S)$ and $A \cup B \in \operatorname{cohesive}(\mathcal{F})$, then by Proposition $3.8 A, B \in$ cohesive $(\mathcal{F})$. Suppose to the contrary that $(A, B) \in$ promise $(\mathcal{F})$. Then $Q \in \mathcal{F}^{\text {dc }}$ exists with $A \subseteq Q$ and $B \subseteq Q^{\mathbf{c}}$. But then $A \subseteq(A \cup B) \cap Q \notin \operatorname{fin}(S)$ and $B \subseteq(A \cup B) \cap Q^{\mathbf{c}} \notin \operatorname{fin}(S)$. This contradicts $A \cup B \in$ cohesive $(\mathcal{F})$.

$(2) \Rightarrow(1)$. Let $A, B \in$ cohesive $(\mathcal{F})$ and $(A, B) \notin$ promise $(\mathcal{F})$. Suppose that $A \cup B \notin$ cohesive $(\mathcal{F})$, i.e. a $Q \in \mathcal{F}^{\text {dc }}$ exists with $(A \cup B) \cap Q,(A \cup$ $B) \cap Q^{\mathbf{c}} \notin \operatorname{fin}(S)$. Let $A_{1}=A \cap Q, B_{1}=B \cap Q, A_{2}=A \cap Q^{\mathbf{c}}$ and $B_{2}=B \cap Q^{\mathbf{c}}$. Then we get the following two cases:

Case 1. $A_{1,2} \notin \operatorname{fin}(S)$ or $B_{1,2} \notin \operatorname{fin}(S)$. Then $A=A_{1} \cup A_{2} \notin \operatorname{cohesive}(\mathcal{F})$ or $B=B_{1} \cup B_{2} \notin$ cohesive $(\mathcal{F})$ - a contradiction.

Case 2. $A_{1}, B_{2} \notin f i n(S)$ and $A_{2}, B_{1} \in \operatorname{fin}(S)$ or $A_{2}, B_{1} \notin f i n(S)$ and $A_{1}, B_{2} \in$ fin $(S)$. Since $\left(A_{1}, B_{2}\right),\left(A_{2}, B_{1}\right) \in \operatorname{promise}(\mathcal{F})$, we can apply Proposition 5.1(8) for $\mathcal{V}=\operatorname{fin}(S)$ and obtain $(A, B) \in \operatorname{promise}(\mathcal{F})$ - a contradiction, again.

We can now characterize those $A, B \in \operatorname{cohesive}(\mathcal{F})$ with $A \cup B \in \operatorname{cohesive}(\mathcal{F})$.

Theorem 6.2. If $\mathcal{F}$ is closed under finite variation and $A, B \in \operatorname{cohesive}(\mathcal{F})$, then the following statements are equivalent:

(1) $A \cup B \in \operatorname{cohesive}(\mathcal{F})$

(2) $(A \backslash B, B) \notin \operatorname{promise}(\mathcal{F})$ or $A \cap B \notin \operatorname{fin}(S)$.

Proof.

$(1) \Rightarrow(2)$ Let $A \cup B \in \operatorname{cohesive}(\mathcal{F})$ and suppose $A \cap B \in \operatorname{fin}(S)$. Then $A \backslash B, B \notin$ fin $(S)$ and $A \backslash B, B \in$ cohesive $(\mathcal{F})$ by Proposition 5.1(8). Clearly, $A \cup B=(A \backslash B) \cup B$ and $A \backslash B \cap B=\emptyset$. Hence by Theorem 6.1 $(A \backslash B, B) \notin$ promise $(\mathcal{F})$.

$(2) \Rightarrow(1)$ Conversely, we have to consider two cases. First suppose that $A \cap B \in$ fin $(S)$ and $(A \backslash B, B) \notin$ promise $(\mathcal{F})$. By assumption and Proposition $3.8 A \backslash B, B \in \operatorname{cohesive}(\mathcal{F})$. Hence, $A \cup B=A \backslash B \cup B \in$ cohesive $(\mathcal{F})$ by Theorem 6.1 . If $A \cap B \notin \operatorname{fin}(S)$, then by Lemma 3.9 $A \cup B \in \operatorname{cohesive}(\mathcal{F})$.

Theorem 6.1 deals essentially with "unsolvability cores" of promise problems for $\mathcal{F}$. This leads to the following definition:

Definition 6.3. $(A, B)$ is a core of $\mathcal{F}((A, B) \in \operatorname{core}(\mathcal{F}))$ if and only if $A, B \notin$ $\operatorname{fin}(S), A \cap B=\emptyset$ and for all $A^{\prime} \subseteq A, B^{\prime} \subseteq B, A^{\prime}, B^{\prime} \notin \operatorname{fin}(S): \quad\left(A^{\prime}, B^{\prime}\right) \notin$ $\operatorname{promise}(\mathcal{F})$. 


\section{Proposition 6.4.}

(1) $\operatorname{core}(\mathcal{F})=\operatorname{core}\left(\mathcal{F}^{c o}\right)=\operatorname{core}\left(\mathcal{F}^{d c}\right)$

(2) $(A, B) \in \operatorname{core}(\mathcal{F}) \Leftrightarrow(B, A) \in \operatorname{core}(\mathcal{F})$.

(3) $(A, B) \in \operatorname{core}(\mathcal{F}), A^{\prime} \subseteq A, B^{\prime} \subseteq B$ and $A^{\prime}, B^{\prime} \notin \operatorname{fin}(S)$ $\Rightarrow\left(A^{\prime}, B^{\prime}\right) \in \operatorname{core}(\mathcal{F})$.

Now, we want to show, that the condition " $A \cup B$ is $\mathcal{F}$-cohesive" characterizes completely the cores of $\mathcal{F}$. The following lemma offers a property of cores, which is similar to the definition of cohesive sets (Def. 3.1).

Lemma 6.5. If $A, B \notin \operatorname{fin}(S)$ and $A \cap B=\emptyset$ then the following statements are equivalent:

(1) $(A, B) \in \operatorname{core}(\mathcal{F})$

(2) $\forall Q \in \mathcal{F}^{d c}:\left(A \cap Q \notin \operatorname{fin}(S) \Leftrightarrow B \cap Q^{c} \in \operatorname{fin}(S)\right)$

Proof.

$(1) \Rightarrow(2)$. Let $(A, B) \in \operatorname{core}(\mathcal{F})$. Consider $Q \in \mathcal{F}^{\mathrm{dc}}$ with $A^{\prime}=A \cap Q \notin \operatorname{fin}(S)$. Suppose to the contrary that $B^{\prime}=B \cap Q^{\mathbf{c}} \notin \operatorname{fin}(S)$. Then $\left(A^{\prime}, B^{\prime}\right) \in$ promise $(\mathcal{F})$, since $A^{\prime} \subseteq Q$ and $B^{\prime} \subseteq Q^{\mathbf{c}}$. This contradicts $(A, B) \in$ core $(\mathcal{F})$. Conversely, let $B \cap Q^{\mathbf{c}} \in \operatorname{fin}(S)$ and suppose $A \cap Q \in \operatorname{fin}(S)$. Then $A^{\prime}=A \cap Q^{\mathbf{c}}$ and $B^{\prime}=B \cap Q$ are infinite. Furthermore $A^{\prime} \subseteq$ $Q^{\mathbf{c}}$ and $B^{\prime} \subseteq Q$. Hence, $\left(A^{\prime}, B^{\prime}\right) \in \operatorname{promise}(\mathcal{F})$ and we get again a contradiction.

$(2) \Rightarrow(1)$. Let the equivalence be valid for any $Q \in \mathcal{F}^{\mathbf{d c}}$. Suppose to the contrary that $(A, B) \notin \operatorname{core}(\mathcal{F})$. Then $A^{\prime} \subseteq A, B^{\prime} \subseteq B$ exist with $A^{\prime}, B^{\prime} \notin$ $\boldsymbol{f i n}(S)$ and $\left(A^{\prime}, B^{\prime}\right) \in$ promise $(\mathcal{F})$. Hence, we can find $Q \in \mathcal{F}^{\text {dc }}$ with $A^{\prime} \subseteq Q, B^{\prime} \subseteq Q^{\mathbf{c}}$. But then $A^{\prime} \subseteq A \cap Q$ and $B^{\prime} \subseteq B \cap Q^{\mathbf{c}}$, i.e. $A \cap Q, B \cap Q^{\mathbf{c}} \notin \operatorname{fin}(S)$ - a contradiction to the equivalence.

Remark 6.6. By Proposition 6.4(1) Lemma 6.5(2) is equivalent to: $\forall Q \in \mathcal{F}^{\text {dc }}$ : $\left(B \cap Q \notin \operatorname{fin}(S) \Leftrightarrow A \cap Q^{\mathbf{c}} \in \operatorname{fin}(S)\right)$.

Theorem 6.7. If $\mathcal{F}$ is closed under finite variation, $A \cap B=\varnothing$ and $A, B \notin \operatorname{fin}(S)$, then the following statements are equivalent:

(1) $(A, B) \in \operatorname{core}(\mathcal{F})$

(2) $A \cup B \in \operatorname{cohesive}(\mathcal{F})$

Proof.

$(1) \Rightarrow(2)$. Let $(A, B) \in \operatorname{core}(\mathcal{F})$. Consider $Q \in \mathcal{F}^{\text {dc }}$ with $(A \cup B) \cap Q \notin \operatorname{fin}(S)$. Then $A \cap Q \notin \operatorname{fin}(S)$ or $B \cap Q \notin \operatorname{fin}(S)$. If $A \cap Q$ is finite, $B \cap Q$ must be infinite. But then $A \cap Q^{\mathbf{c}} \in \operatorname{fin}(S)$ by Lemma 6.5 and therefore $A \in \boldsymbol{f i n}(S)$, which contradicts the assumption $A \notin \operatorname{fin}(S)$. Hence, $A \cap Q$ must be infinite. By the same reason $B \cap Q$ must be infinite, too. But then $B \cap Q^{\mathbf{c}}, A \cap Q^{\mathbf{c}} \in \operatorname{fin}(S)$ by Lemma 6.5 and therefore $(A \cup B) \cap Q^{\mathbf{c}} \in \operatorname{fin}(S)$. In total $A \cup B \in \operatorname{cohesive}(\mathcal{F})$. 
$(2) \Rightarrow(1)$. Suppose that $A \cup B \in$ cohesive $(\mathcal{F})$. Let $A^{\prime} \subseteq A, B^{\prime} \subseteq B$ such that $A^{\prime}, B^{\prime} \notin \operatorname{fin}(S)$. Then $A^{\prime} \cap B^{\prime}=\emptyset$ and $A^{\prime} \cup B^{\prime} \subseteq A \cup B$, i.e. $A^{\prime} \cup B^{\prime} \in$ cohesive $(\mathcal{F})$. But then $\left(A^{\prime}, B^{\prime}\right) \notin$ promise $(\mathcal{F})$ by Theorem 6.1 and therefore $(A, B) \in \operatorname{core}(\mathcal{F})$.

Corollary 6.8. If $\mathcal{F}$ is closed under finite variation, $A, B, C \notin \operatorname{fin}(S), B \subseteq C$ and $A \cap C=\varnothing$, then $((A, B) \in \operatorname{core}(\mathcal{F})$ and $C \in \operatorname{cohesive}(\mathcal{F}))$ implies $(A, C) \in$ $\operatorname{core}(\mathcal{F})$.

Proof.

Let $A, B, C$ be given according to the assumption. Consider $(A, B) \in \operatorname{core}(\mathcal{F})$ and $C \in \operatorname{cohesive}(\mathcal{F})$. Then $A \cup B \in \operatorname{cohesive}(\mathcal{F})$ by Theorem 6.7. Since $(A \cup B) \cap C=$ $B \notin \operatorname{fin}(S)$, we get $(A \cup B) \cup C=A \cup C \in \operatorname{cohesive}(\mathcal{F})$ by Lemma 3.9. In conclusion $(A, C) \in \operatorname{core}(\mathcal{F})$ by Theorem 6.7 .

Moreover, we obtain the transitivity of cores.

Corollary 6.9. If $\mathcal{F}$ is closed under finite variation and $A \cap B=A \cap C=B \cap C=$ $\emptyset$, then $((A, B) \in \operatorname{core}(\mathcal{F})$ and $(B, C) \in \operatorname{core}(\mathcal{F}))$ implies $(A, C) \in \operatorname{core}(\mathcal{F})$.

Proof.

Let $A, B, C$ be given according to the assumption. Let $(A, B) \in \operatorname{core}(\mathcal{F})$ and $(B, C) \in \operatorname{core}(\mathcal{F})$. Then $B \cup C \in \operatorname{cohesive}(\mathcal{F})$ by Theorem 6.7. Hence $C \in$ cohesive $(\mathcal{F})$ by Proposition 3.8. But now, $(A, B \cup C) \in \operatorname{core}(\mathcal{F})$ by Corollary 6.8 and therefore $(A, C) \in \operatorname{core}(\mathcal{F})$ by Proposition $6.4(2)$.

Combining Theorem 6.7 with Lemma 3.4 we get

Corollary 6.10. If $\mathcal{F}$ is closed under finite variation and $\mathcal{F}=\mathcal{F}^{c c}$, then $\operatorname{core}(\mathcal{F})=\operatorname{core}\left(\mathcal{F}^{b}\right)$.

In contrast to the transitivity of cores, the property not belonging to promise $(\mathcal{F})$ is transitive only with an additional condition.

Lemma 6.11. Let $\mathcal{F}$ be closed under finite variation and $A \cap B=A \cap C=B \cap C=$ $\emptyset$. If $B \in \operatorname{cohesive}(\mathcal{F})$, then $(A, B) \notin \operatorname{promise}(\mathcal{F})$ and $(B, C) \notin \operatorname{promise}(\mathcal{F})$ implies $(A, C) \notin$ promise $(\mathcal{F})$.

Proof.

Suppose that $(A, B) \notin$ promise $(\mathcal{F}),(B, C) \notin$ promise $(\mathcal{F})$ and $(A, C) \in$ promise $(\mathcal{F})$. Let $Q \in \mathcal{F}^{\text {dc }}$ with $A \subseteq Q$ and $C \subseteq Q^{\mathbf{c}}$. Since $B \in \operatorname{cohesive}(\mathcal{F})$, $B \cap Q$ or $B \cap Q^{\mathbf{c}}$ has to be finite. By symmetry we can assume, that $D=B \cap Q \in$ fin $(S)$. But then, $A \cup D \subseteq Q$ and $C \cup\left(B \cap D^{\mathbf{c}}\right) \subseteq Q^{\mathbf{c}}$, i.e. $\left(A \cup D, C \cup\left(B \cap D^{\mathbf{c}}\right)\right) \in$ promise $(\mathcal{F})$. Thus, $\left(A \cup D, B \cap D^{\mathbf{c}}\right) \in \operatorname{promise}(\mathcal{F})$, too and we can apply Proposition 5.1(8) and conclude, that $(A, B) \in \operatorname{promise}(\mathcal{F})$ - a contradiction. 
As shown in [1] by a marking technique this kind of transitivity is not valid in the general case. The same technique is used in

Example 6.12. Let $X=\{a, b\}$. Consider a language family $\mathcal{L}$ satisfying the condition of Lemma 5.4. Choose $A$ with $A, A^{\mathbf{c}} \notin \mathcal{L}$. Then $\left(A, A^{\mathbf{c}}\right) \notin$ promise $(\mathcal{L})$ and by Lemma $5.4\left(x A, x A^{\mathbf{c}}\right) \notin \operatorname{promise}(\mathcal{L})$ for any $x \in X$. Hence $\left(a A, a A^{\mathbf{c}} \cup\right.$ $\left.b A^{\mathbf{c}}\right),\left(a A^{\mathbf{c}} \cup b A^{\mathbf{c}}, b A\right) \notin$ promise $(\mathcal{L})$. But $(a A, b A) \in$ promise $(\mathcal{L})$.

Next we want to show, that under some closure condition for $\mathcal{F}$ any $(A, B) \notin$ promise $(\mathcal{F})$ contains a core of $\mathcal{F}$. We shall use a construction similar to the construction in the proof of the Dekker-Myhill theorem. For this purpose the following lemma is crucial.

Lemma 6.13. If $\mathcal{F}^{u}=\mathcal{F}=\mathcal{F}^{s}$ and $(A, B) \notin$ promise $(\mathcal{F})$, then for all $Q \in \mathcal{F}^{\text {dc }}$ : $(A \cap Q, B \cap Q) \notin \operatorname{promise}(\mathcal{F})$ or $\left(A \cap Q^{c}, B \cap Q^{c}\right) \notin \operatorname{promise}(\mathcal{F})$.

\section{Proof.}

Suppose to the contrary a $Q \in \mathcal{F}^{\mathrm{dc}}$ exists, such that $(A \cap Q, B \cap Q) \in \operatorname{promise}(\mathcal{F})$ and $\left(A \cap Q^{\mathbf{c}}, B \cap Q^{\mathbf{c}}\right) \in$ promise $(\mathcal{F})$. Then we can find $Q_{1,2} \in \mathcal{F}^{\text {dc }}$ with $A \cap Q \subseteq$ $Q_{1}, B \cap Q \subseteq Q_{1}^{\mathbf{c}}$ and $A \cap Q^{\mathbf{c}} \subseteq Q_{2}, B \cap Q^{\mathbf{c}} \subseteq Q_{2}^{\mathbf{c}}$. Now, $A=(A \cap Q) \cup\left(A \cap Q^{\mathbf{c}}\right) \subseteq$ $\left(Q_{1} \cap Q\right) \cup\left(Q_{2} \cap Q^{\mathbf{c}}\right)$ and $B=(B \cap Q) \cup\left(B \cap Q^{\mathbf{c}}\right) \subseteq\left(Q_{1}^{\mathbf{c}} \cap Q\right) \cup\left(Q_{2}^{\mathbf{c}} \cap Q^{\mathbf{c}}\right)$. Let $Q_{A}=\left(Q_{1} \cap Q\right) \cup\left(Q_{2} \cap Q^{\mathbf{c}}\right)$ and $Q_{B}=\left(Q_{1}^{\mathbf{c}} \cap Q\right) \cup\left(Q_{2}^{\mathbf{c}} \cap Q^{\mathbf{c}}\right)$. Then $Q_{A} \cup Q_{B}=$ $Q \cup Q^{\mathbf{c}}=S$ and $Q_{A} \cap Q_{B}=\emptyset$. Hence, $Q_{B}=Q_{A}^{\mathbf{c}}$. Since $A \subseteq Q_{A}, B \subseteq Q_{B}=Q_{A}^{\mathbf{c}}$ and $Q_{A}, Q_{B} \in \mathcal{F}$ we get by the closure properties of $\mathcal{F}$ in total $(A, B) \in \operatorname{promise}(\mathcal{F})$ - a contradiction.

Theorem 6.14. If $\mathcal{F}$ is denumerable, closed under finite variation and $\mathcal{F}^{u}=$ $\mathcal{F}=\mathcal{F}^{s}$, then for all $(A, B)$ with $A \cap B=\emptyset$ and $(A, B) \notin \operatorname{promise}(\mathcal{F})$, there exist $A^{\prime} \subseteq A, B^{\prime} \subseteq B$, such that $\left(A^{\prime}, B^{\prime}\right) \in \operatorname{core}(\mathcal{F})$.

Proof.

Let $\mathbf{e}_{\mathcal{F}}: \mathbb{N}_{0} \rightarrow \mathbf{2}^{S}$ with $\mathbf{e}_{\mathcal{F}}\left(\mathbb{N}_{0}\right)=\mathcal{F}$ and $(A, B) \notin$ promise $(\mathcal{F})$. Then we construct the following sequence of pairs $\left(A_{n}, B_{n}\right)$ for $n \geq 0$ inductively by

$$
\begin{aligned}
& \left(A_{0}, B_{0}\right)=(A, B) \\
& \left(A_{n+1}, B_{n+1}\right)=\text { if }\left(A_{n} \cap \mathbf{e}_{\mathcal{F}}(n), B_{n} \cap \mathbf{e}_{\mathcal{F}}(n)\right) \notin \operatorname{promise}(\mathcal{F}) \\
& \text { then }\left(A_{n} \cap \mathbf{e}_{\mathcal{F}}(n), B_{n} \cap \mathbf{e}_{\mathcal{F}}(n)\right) \\
& \text { else }\left(A_{n} \cap \mathbf{e}_{\mathcal{F}}(n)^{\mathbf{c}}, B_{n} \cap \mathbf{e}_{\mathcal{F}}(n)^{\mathbf{c}}\right) \mathbf{f i .} .
\end{aligned}
$$

Assertion 1. $\forall n \geq 0: A_{n+1} \subseteq A_{n}, B_{n+1} \subseteq B_{n}$ and $\left(A_{n}, B_{n}\right) \notin \operatorname{promise}(\mathcal{F})$.

Clearly, $A_{n+1} \subseteq A_{n}$ and $B_{n+1} \subseteq B_{n}$ for $n \geq 0$ follows directly from the definition. The second part of the assertion is proven by induction on $n$. If $n=0$ then $\left(A_{0}, B_{0}\right)=(A, B) \notin$ promise $(\mathcal{F})$ by assumption. Consider $\left(A_{n+1}, B_{n+1}\right)$. If $\left(A_{n} \cap \mathbf{e}_{\mathcal{F}}(n), B_{n} \cap \mathbf{e}_{\mathcal{F}}(n)\right) \notin \operatorname{promise}(\mathcal{F})$, nothing is to prove. Suppose $\left(A_{n} \cap \mathbf{e}_{\mathcal{F}}(n), B_{n} \cap \mathbf{e}_{\mathcal{F}}(n)\right) \in \operatorname{promise}(\mathcal{F})$. Since $\mathcal{F}^{\mathbf{u}}=\mathcal{F}=\mathcal{F}^{\mathbf{s}}$, we get by Lemma $5.12\left(A_{n+1}, B_{n+1}\right)=\left(A_{n} \cap \mathbf{e}_{\mathcal{F}}(n)^{\mathbf{c}}, B_{n} \cap \mathbf{e}_{\mathcal{F}}(n)^{\mathbf{c}}\right) \notin \operatorname{promise}(\mathcal{F})$. 
Assertion 2. $\forall n \geq 0 \exists k \geq n: A_{k} \subset A_{n}$ and $B_{k} \subset B_{n}$.

Assume $n \geq 0$ exists with $A_{j}=A_{n}$ for all $j \geq n$. Since $A_{n} \notin \operatorname{fin}(S), x, y \in$ $A_{n}$ exist with $x \neq y$. Since $\mathcal{F}$ is closed under finite variation, $x \in \mathbf{e}_{\mathcal{F}}(m)$ and $y \in \mathbf{e}_{\mathcal{F}}(m)^{\mathbf{c}}$ for some $m$. Furthermore $m \geq n$, otherwise $x$ and $y$ can not be both in $A_{n}$. Hence, by construction either $x \notin A_{m+1}$ or $y \notin A_{m+1}$, i.e. $A_{m+1} \neq A_{n}$, while on the other side by our assumption $A_{m+1}=A_{m}=A_{n}$ - a contradiction. Analogously, $m^{\prime} \geq n$ exists with $B_{m^{\prime}} \subset B_{n}$. Choosing $k=\boldsymbol{m a x}\left(m, m^{\prime}\right)$ we get the result by ass.1.

On the basis of ass.2 a function $g: \mathbb{N}_{0} \rightarrow \mathbb{N}_{0}$ exists with $A_{g(i+1)} \subset A_{g(i)} \subset A_{i}$ and $B_{g(i+1)} \subset B_{g(i)} \subset B_{i}$ for any $i \geq 0$. But then two sequences $a_{i}$ and $b_{i}$ exist with $a_{i} \in A_{g(i)} \backslash A_{g(i+1)}$ and $b_{i} \in B_{g(i)} \backslash B_{g(i+1)}(i \geq 0)$ and the property: $0 \leq i<j \Rightarrow a_{i} \neq a_{j}$ and $b_{i} \neq b_{j}$. Hence, the two sets $A^{\prime}=\left\{a_{i} \mid i \geq 0\right\}$ and $B^{\prime}=\left\{b_{i} \mid i \geq 0\right\}$ are both infinte. Furthermore, $A^{\prime} \cap B^{\prime}=\emptyset$, since $A^{\prime} \subseteq A, B^{\prime} \subseteq B$ and $A \cap B=\emptyset$.

Now, we can show that $A^{\prime} \cup B^{\prime} \in \operatorname{cohesive}(\mathcal{F})$. Then $A^{\prime} \cup B^{\prime} \in \operatorname{core}(\mathcal{F})$ follows directly by Theorem 6.7 completing the proof of the theorem.

Consider $Q \in \mathcal{F}^{\mathbf{d c}}$, i.e. $Q=\mathbf{e}_{\mathcal{F}}(m)$ for some $m \geq 0$. Then $\left(A_{m+1}, B_{m+1}\right)=$ $\left(A_{m} \cap \mathbf{e}_{\mathcal{F}}(m), B_{m} \cap \mathbf{e}_{\mathcal{F}}(m)\right)$ or $\left(A_{m+1}, B_{m+1}\right)=\left(A_{m} \cap \mathbf{e}_{\mathcal{F}}(m)^{\mathbf{c}}, B_{m} \cap \mathbf{e}_{\mathcal{F}}(m)^{\mathbf{c}}\right)$. Hence, by definition of $g$ :

(1) $A_{g(m+1)} \cup B_{g(m+1)} \subset A_{m+1} \cup B_{m+1} \subset \mathbf{e}_{\mathcal{F}}(m)$ or

(2) $A_{g(m+1)} \cup B_{g(m+1)} \subset A_{m+1} \cup B_{m+1} \subset \mathbf{e}_{\mathcal{F}}(m)^{\mathbf{c}}$.

Consider the first case. Observe that $A^{\prime} \cap A_{g(k)}=\left\{a_{i} \mid i \geq k\right\}$ and $B^{\prime} \cap B_{g(k)}=$ $\left\{b_{i} \mid i \geq k\right\}$, i.e. almost all $a_{i}$ belong to $A^{\prime} \cap A_{g(k)}$ and almost all $b_{i}$ belong to $B^{\prime} \cap B_{g(k)}$ for any $k \geq 0$. Noticing $A^{\prime} \cap B=\emptyset=A \cap B^{\prime}$, we get

$$
\begin{aligned}
\left(A^{\prime} \cup B^{\prime}\right) \cap Q & =\left(A^{\prime} \cup B^{\prime}\right) \cap \mathbf{e}_{\mathcal{F}}(m) \\
& =\left(A^{\prime} \cup B^{\prime}\right) \cap\left(A_{g(m+1)} \cup B_{g(m+1)}\right) \\
& =\left(A^{\prime} \cap A_{g(m+1)}\right) \cup\left(B^{\prime} \cap B_{g(m+1)}\right) \\
& =\left(A^{\prime} \cap B^{\prime}\right) \backslash C
\end{aligned}
$$

for some finite set $C$, i.e. $\left(A^{\prime} \cup B^{\prime}\right) \cap Q^{\mathbf{c}} \in \operatorname{fin}(S)$. Completely analogously, we find in the second case $\left(A^{\prime} \cup B^{\prime}\right) \cap Q \in \boldsymbol{f i n}(S)$. Hence, $A^{\prime} \cup B^{\prime} \in \operatorname{cohesive}(\mathcal{F})$.

Corollary 6.15. If $\mathcal{F}$ is denumerable, closed under finite variation and $\mathcal{F}=\mathcal{F}^{c c}$, then for all $(A, B) \notin$ promise $(\mathcal{F})$, there exist $A^{\prime} \subseteq A, B^{\prime} \subseteq B$ with $\left(A^{\prime}, B^{\prime}\right) \in$ $\operatorname{core}(\mathcal{F})$.

Proof.

Since $\operatorname{core}(\mathcal{F})=\operatorname{core}\left(\mathcal{F}^{\mathbf{b}}\right)$ by Corollary 6.10 , the conditions of Theorem 6.14 are met for $\mathcal{F}^{\mathbf{b}}$. Hence, we find $A^{\prime} \subseteq A, B^{\prime} \subseteq B$ with $\left(A^{\prime}, B^{\prime}\right) \in \operatorname{core}\left(\mathcal{F}^{\mathbf{b}}\right)=$ $\operatorname{core}(\mathcal{F})$.

The following example shows, that the closure conditions of Theorem 6.14 respectively Corollary 6.15 are necessary. If we look for example at the family of 
contextfree languages which is closed neither under intersection nor under complement, we find unsolvable promise problems without cores.

Example 6.16. Consider $X=\{a, b, c\}$. For $x \in X$ and $w \in X^{*}$ let $|w|_{x}$ denote the number of occurences of $x$ in $w$. Then define for $x, y \in X$ with $x \neq y: L_{x, y}=\{w \in$ $\left.\left.X^{*}|| w\right|_{x} \neq|w|_{y}\right\} . L_{x, y} \in \mathcal{L}_{\mathbf{c f}}(X)$, moreover, $L_{x, y}$ is a deterministic contextfree language, hence $L_{x, y}^{\mathbf{c}} \in \mathcal{L}_{\mathbf{c f}}(X)$. Consider $A=L_{a, b} \cup L_{b, c} \cup L_{c, a}$ and $B=A^{\mathbf{c}}=\{w \in$ $\left.X^{*} \|\left. w\right|_{a}=|w|_{b}=|w|_{c}\right\}=L_{a, b}^{\mathbf{c}} \cap L_{b, c}^{\mathbf{c}} \cap L_{c, a}^{\mathbf{c}}$. Then $A \in \mathcal{L}_{\mathbf{c f}}(X), B \notin \mathcal{L}_{c f}(X), B \in$ $\mathcal{L}_{\mathbf{c f}}(X)^{\text {co }}$ and $B \in \mathcal{L}_{\mathbf{c f}}(X)^{\mathbf{s}}$. This shows $(A, B) \notin \operatorname{promise}\left(\mathcal{L}_{\mathbf{c f}}(X)\right),(A, B) \in$ promise $\left(\mathcal{L}_{\mathbf{c f}}(X)^{\text {co }}\right)$ and $(A, B) \in \operatorname{promise}\left(\mathcal{L}_{\mathbf{c f}}(X)^{\mathbf{s}}\right)$.

Now, suppose $A^{\prime}, B^{\prime} \notin \operatorname{fin}(S)$ exist with $A^{\prime} \subseteq A, B^{\prime} \subseteq B$ and $\left(A^{\prime}, B^{\prime}\right) \in$ $\operatorname{core}\left(\mathcal{L}_{\text {cf }}(X)\right)$. Since $A^{\prime}$ is infinite, $A^{\prime} \cap L_{x, y}$ is infinite for at least one of the pairs $(x, y)$. Assume without loss of generality $x=a$ and $y=b$. But then, $\left(A^{\prime} \cap L_{a, b}, B^{\prime}\right) \in \operatorname{core}\left(\mathcal{L}_{\mathbf{c f}}(X)\right)$ and therefore $\left(A^{\prime} \cap L_{a, b}, B^{\prime}\right) \notin \operatorname{promise}\left(\mathcal{L}_{\mathbf{c f}}(X)\right)$. On the other side, $A^{\prime} \cap L_{a, b} \subseteq L_{a, b}$ and $B^{\prime} \subseteq B \subseteq L_{a, b}^{\mathbf{c}}$, i.e. $\left(A^{\prime} \cap L_{a, b}, B^{\prime}\right) \in$ promise $\left(\mathcal{L}_{\mathbf{c f}}(X)\right)$ and we get a contradiction.

We get one further corollary of Theorem 6.14. For $\mathcal{F}$ and $A \notin \operatorname{fin}(S)$ define $\operatorname{core}(A, \mathcal{F})=\left\{B \mid B \notin \operatorname{fin}(S), A \cap B=\emptyset\right.$ and $\forall B^{\prime} \subseteq B, B^{\prime} \notin \operatorname{fin}(S):\left(A, B^{\prime}\right) \notin$ $\operatorname{promise}(\mathcal{F})\}$.

Corollary 6.17. If $\mathcal{F}$ is denumerable, closed under finite variation and $\mathcal{F}^{u}=$ $\mathcal{F}=\mathcal{F}^{s}$, then for all $A, A^{c} \notin \mathcal{F} \cup \operatorname{fin}(S)^{c c}: \operatorname{core}(A, \mathcal{F}) \neq \varnothing$.

Proof.

By Proposition 5.1(4) $\left(A, A^{\mathbf{c}}\right) \notin$ promise $(\mathcal{F})$. By Theorem $6.14 A^{\prime} \subseteq A, B^{\prime} \subseteq A^{\mathbf{c}}$ exist with $\left(A^{\prime}, B^{\prime}\right) \in \operatorname{core}(\mathcal{F})$. Then for any $B^{\prime \prime} \subseteq B$ with $B^{\prime \prime} \notin \operatorname{fin}(S),\left(A^{\prime}, B^{\prime \prime}\right) \notin$ $\operatorname{promise}(\mathcal{F})$ and therefore $\left(A, B^{\prime \prime}\right) \notin \operatorname{promise}(\mathcal{F})$.

\section{COMplexity CORES}

We have seen that under the conditions of Corollary $6.17 \operatorname{core}(A, \mathcal{F}) \neq \varnothing$, provided there exists a $B$ with $(A, B) \notin \operatorname{promise}(\mathcal{F})$. We can improve the result under the same assumption by connecting the elements of $\operatorname{core}(A, \mathcal{F})$ to the hard cores (of complexity classes) introduced in a general form by Book-Du [2]. For $\mathcal{F}$ and $A$ define $\mathcal{F}(A)=\{Q \in \mathcal{F} \mid Q \subseteq A\}$.

Definition 7.1 (see [2]). $B$ is an $\mathcal{F}$-hardcore of $A$ if and only if $B \notin \operatorname{fin}(S)$ and for all $C \in \mathcal{F}(A): B \cap C \in \operatorname{fin}(S)$. If additionally $B \subseteq A$, then $B$ is a proper $\mathcal{F}$-hardcore of $A$.

Lemma 7.2. If $\mathcal{F}$ is closed under finite variation with $\mathcal{F}=\mathcal{F}^{c c}$ and $A \cap B=\varnothing$, then

(1) $(A, B) \notin$ promise $(\mathcal{F}) \Leftrightarrow B^{c} \in \operatorname{immune}\left(\mathcal{F}\left(A^{c}\right)^{c o}\right)$.

(2) $B \in \operatorname{core}(A, \mathcal{F}) \Leftrightarrow B$ is a proper $\mathcal{F}$-hardcore of $A^{c}$. 
Proof. We make use of the (trivial)

Assertion. $A \subseteq Q$ and $Q \in \mathcal{F} \Leftrightarrow Q \in \mathcal{F}\left(A^{\text {c }}\right)^{\text {co }}$.

(1) Suppose $(A, B) \notin$ promise $(\mathcal{F})$ and $B^{\mathbf{c}} \notin$ immune $\left(\mathcal{F}\left(A^{\mathbf{c}}\right)^{\mathbf{c o}}\right)$. Then $Q \in$ $\mathcal{F}\left(A^{\mathbf{c}}\right)^{\text {co }}$ exists with $Q \notin \operatorname{fin}(S)$ and $Q \subseteq B^{\mathbf{c}}$. But then $B \subseteq Q Q^{\mathbf{c}}, A \subseteq Q$ and $Q \in \mathcal{F}^{\mathrm{dc}}-$ a contradiction.

Conversely, if $(A, B) \in$ promise $(\mathcal{F})$, a $Q \in \mathcal{F}^{\text {dc }}$ exists with $A \subseteq Q, B \subseteq Q^{\mathbf{c}}$. But then, $Q \in \mathcal{F}\left(A^{\mathbf{c}}\right)^{\text {co }}$ and $Q \subseteq B^{\mathbf{c}}$. Since $A \notin \operatorname{fin}(S), Q \notin \operatorname{fin}(S)$, i.e. $B^{\mathbf{c}} \notin \operatorname{immune}\left(\mathcal{F}\left(A^{\mathrm{c}}\right)^{\mathrm{co}}\right)$.

(2) Consider $B \in \operatorname{core}(A, \mathcal{F})$. Then by definition $B \notin \operatorname{fin}(S)$ and $B \subseteq A^{\mathbf{c}}$. Now, suppose that $B$ is not a proper $\mathcal{F}$-hardcore of $A^{\mathbf{c}}$. Then $Q \in \mathcal{F}\left(A^{\mathbf{c}}\right)$ exists with $B \cap Q \notin \operatorname{fin}(S)$. Clearly, $B \cap Q \subseteq Q \subseteq A^{\mathbf{c}}$. Moreover, $A \subseteq Q^{\mathbf{c}}$ and $Q \in \mathcal{F}=\mathcal{F}^{\text {dc }}$. Hence $(A, B \cap Q) \in \operatorname{promise}(\mathcal{F})$, a contradiction to $B \in \operatorname{core}(A, \mathcal{F})$.

Conversely, consider a proper $\mathcal{F}$-hardcore $B$ of $A^{\text {c }}$ and suppose, that $B \notin$ $\operatorname{core}(A, \mathcal{F})$, i.e. $\left(A, B^{\prime}\right) \in \operatorname{promise}(\mathcal{F})$ for some $B^{\prime} \subseteq B$ with $B^{\prime} \notin \operatorname{fin}(S)$. Then $Q \in \mathcal{F}^{\text {dc }}$ exists with $A \subseteq Q$ and $B \subseteq Q^{\mathbf{c}}$. Since $B^{\prime} \subseteq B \cap Q^{\mathbf{c}} \notin$ $\operatorname{fin}(S)$ and $B \cap Q^{\mathbf{c}} \subseteq A^{\mathbf{c}}, B$ is not a proper $\mathcal{F}$-hardcore of $A^{\mathbf{c}}$ and we get a contradiction.

In [2] Book and Du characterize hard cores in the following way:

Theorem $7.3([2])$. Let $\mathcal{F}$ be a denumerable set family and $A \notin \operatorname{fin}(S)$. Then a proper $\mathcal{F}$-hardcore $B$ of $A$ exists if and only if $A \notin \mathcal{F}^{u} \oplus \operatorname{fin}(S)$.

Furthermore they achieved the existence of recursive hard cores under the following conditions:

Theorem 7.4 ([2]). If $\mathcal{L}$ is WP-recursive, closed under finite variation and $\mathcal{L}=$ $\mathcal{L}^{u}$, then for any $A \in \mathcal{L}_{\text {rec }}(X) \backslash \mathcal{L}$, a proper $\mathcal{L}$-hardcore $B \in \mathcal{L}_{\text {rec }}(X)$ of $A$ exists.

Combining Lemma 7.2 with Theorem 7.4 we obtain

Theorem 7.5. If $\mathcal{L}$ is $W P$-recursive and $\mathcal{L}=\mathcal{L}^{b}$, then for any $A \in \mathcal{L}_{\text {rec }}(X) \backslash \mathcal{L}$ : $\operatorname{core}(A, \mathcal{F}) \cap \mathcal{L}_{\text {rec }}(X) \neq \emptyset$.

\section{Concluding Remarks}

It is natural, to consider $n$-dimensional promise problems $\left(A_{1}, \ldots, A_{n}\right)$ with $A_{i} \cap A_{j}=\varnothing$ and $A_{i} \subseteq S$ for $1 \leq i \neq j \leq n$. For a set family $\mathcal{F}$ the promise problem is solvable if a partition $\left(Q_{1}, \ldots, Q_{n}\right)$ of $S$ exists with $A_{i} \subseteq Q_{i}, Q_{i} \in$ $\mathcal{F}(1 \leq i \leq n)$. For $n>2$ cores of unsolvability can be characterized by cohesiveness of $A_{1} \cup \ldots \cup A_{n}$, too. But for $n=3$ unsolvable promise problems exist, which have no cores of unsolvability ([1]).

We can strengthen Lemma 4.2 about the structure of cohesive sets using a much smaller family than $\mathcal{L}_{\text {reg }}(X)$. Let $\mathcal{L}_{\text {ltr }}(X)=\left\{w_{1} L_{1} \cup \ldots \cup w_{k} L_{k} \mid k>0, w_{i} \in\right.$ 
$X^{*}, L_{i} \in \operatorname{fin}\left(X^{*}\right)^{\mathbf{c c}}$ for $\left.1 \leq i \leq k\right\}=\left(\left(\operatorname{fin}\left(X^{*}\right)^{\mathbf{c c}}\right)^{\mathbf{l t r}}\right)^{\mathbf{u}}$. Then this lemma holds for $\mathcal{L}_{\text {ltr }}(X)$, too. Hence, Lemma 4.2 is true not only for language families $\mathcal{L}$ containing $\mathcal{L}_{\text {reg }}(X)$, but also for all $\mathcal{L}$ with $\mathcal{L}_{\text {ltr }}(X) \subseteq \mathcal{L} \subseteq \mathcal{L}_{\text {reg }}(X)$. Moreover, the variation condition " $\mathcal{L} \pm \mathcal{L}_{\text {reg }}(X) \subseteq \mathcal{L}$ " can be replaced by " $\mathcal{L} \pm \mathcal{L}_{\text {ltr }}(X) \subseteq \mathcal{L}$ " in all results and examples involving the handling of left markers.

\section{REFERENCES}

[1] K. Ambos-Spies, U. Brandt and M. Ziegler, Real Benefit of Promises and Advice, accepted for presentation at CiE 2013 in vol. 7921 of Lect. Notes Comput. Sci. Springer (2013) 1-11.

[2] R.V. Book, D.-Z. Du, The Existence and Density of Generalized Complexity Cores, in J. ACM 34 (1987) 718-730.

[3] J.L. Balcazar, J. Diaz and J. Gabarro, Structural Complexity I, EATCS Monographs on Theoretical Computer Science. Springer Verlag, Heidelberg (1988).

[4] S. Even, A.L. Selman and Y. Yacobi, The Complexity of Promise Problems with Applications to Public-Key Cryptography. Information and Control 61 (1984) 159-173.

[5] O. Goldreich, On Promise-Problems, A Survey in Memory of Shimon Even. Dep. Comp. Science, Weizmann Institute of Science (2005).

[6] M.A. Harrison, Introduction to Formal Language Theory. Addison-Wesley Publishing Company (1978).

[7] G. Rozenberg and A. Salomaa, Word, Language, Grammar, in vol. 1 of Handbook of Formal Languages. Springer Verlag (1997).

[8] H.R. Jun, Theory of Recursive Functions and Effective Computability. MacGraw-Hill Book Company (1967).

[9] R.I. Soare, Recursively enumerable Sets and Degrees. Springer Verlag (1987).

Communicated by V. Diekert.

Received April 14, 2013. Accepted August 22, 2013. 